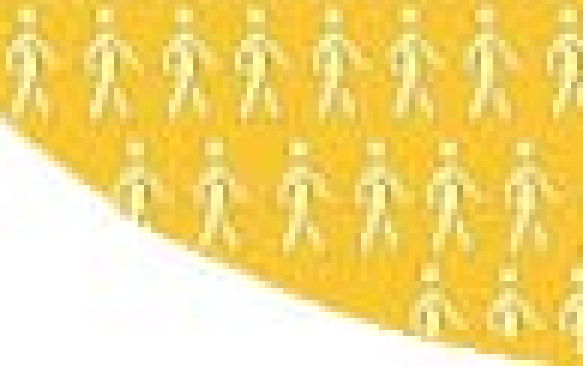

NONPARAMETRIC ESTIMATION OF AN ADDITIVE QUANTILE REGRESSION MODEL

Joel L. Horowitz Sokbae Lee

THE INSTITUTE FOR FISCAL STUDIES DEPARTMENT OF ECONOMICS, UCL cemmap working paper CWP07/04 


\title{
Nonparametric Estimation of an Additive Quantile Regression Model
}

\author{
Joel L. Horowitz \\ Department of Economics \\ Northwestern University \\ Evanston, IL 60208, USA \\ joel-horowitz@northwestern.edu \\ and \\ Sokbae Lee * \\ Centre for Microdata Methods and Practice \\ Institute for Fiscal Studies \\ and \\ Department of Economics \\ University College London \\ London, WC1E 6BT, UK \\ l.simon@ucl.ac.uk
}

December 2004

\begin{abstract}
This paper is concerned with estimating the additive components of a nonparametric additive quantile regression model. We develop an estimator that is asymptotically normally distributed with a rate of convergence in probability of $n^{-r /(2 r+1)}$ when the additive components are $r$-times continuously differentiable for some $r \geq 2$. This result holds regardless of the dimension of the covariates and, therefore, the new estimator has no curse of dimensionality. In addition, the estimator has an oracle property and is easily extended to a generalized additive quantile regression model with a link function. The numerical performance and usefulness of the estimator are illustrated by Monte Carlo experiments and an empirical example.
\end{abstract}

\footnotetext{
${ }^{*}$ We thank Andrew Chesher, Hidehiko Ichimura, Roger Koenker, an editor, an associate editor, and two anonymous referees for helpful comments and suggestions. One referee kindly pointed out some mistakes in the proofs of an earlier draft and suggested corrections. Special thanks go to Andrew Chesher for encouraging us to work on this project. A preliminary version of the paper was presented at the Cemmap Workshop on Quantile Regression Methods and Applications in April, 2003, London, U.K. and at the 2004 Far Eastern Meeting of the Econometric Society, June 30 - July 2, 2004, Seoul, Korea. The research of Joel L. Horowitz was supported in part by NSF Grants SES-9910925 and SES-0352675 and this work was partially supported by ESRC Grant RES-000-22-0704.
} 


\section{Introduction}

This paper is concerned with nonparametric estimation of the functions $m_{1, \alpha}, \ldots, m_{d, \alpha}$ in the quantile regression model

$$
Y=\mu_{\alpha}+m_{1, \alpha}\left(X^{1}\right)+\cdots+m_{d, \alpha}\left(X^{d}\right)+U_{\alpha},
$$

where $Y$ is a real-valued dependent variable, $X^{j}(j=1, \ldots, d)$ is the $j$ 'th component of random vector $\mathbf{X} \in \mathbf{R}^{d}$ for some finite $d \geq 2, \mu_{\alpha}$ is an unknown constant, $m_{1, \alpha}, \ldots, m_{d, \alpha}$ are unknown functions, and $U_{\alpha}$ is an unobserved random variable whose $\alpha$-th quantile conditional on $\mathbf{X}=\mathbf{x}$ is zero for almost every $\mathbf{x}$. Estimation is based on an iid random sample $\left\{\left(Y_{i}, \mathbf{X}_{i}\right): i=1, \ldots, n\right\}$ of $(Y, \mathbf{X})$. We describe an estimator of the additive components

$m_{1, \alpha}, \ldots, m_{d, \alpha}$ that converges in probability pointwise at the rate $n^{-r /(2 r+1)}$ when the $m_{j, \alpha}$ are $r$ times continuously differentiable. This result holds regardless of the dimension of $\mathbf{X}$, so asymptotically there is no curse of dimensionality. Moreover, our estimator has an oracle property. Specifically, the centered, scaled estimator of each additive component is asymptotically normally distributed with the same mean and variance that it would have if the other components were known. Finally, it is straightforward to extend our estimator to the generalized additive model

$$
G(Y)=\mu_{\alpha}+m_{1, \alpha}\left(X^{1}\right)+\cdots+m_{d, \alpha}\left(X^{d}\right)+U_{\alpha},
$$

where $G$ is a known, strictly increasing function.

Additive modeling is an important way to achieve dimension reduction in multivariate nonparametric mean or quantile regression. There are many applications in which simple parametric models fit the available data poorly and, therefore, a more flexible approach to estimation is needed (e.g., Härdle (1990), Horowitz (1993), Horowitz and Lee (2002), and Horowitz and Savin (2001), among many others). Fully nonparametric estimation avoids the problem of poor fit but is usually unattractive in multivariate settings because the curse of dimensionality typically causes fully nonparametric estimates to be very imprecise with samples of the sizes found in applications. Nonparametric additive models reduce the effective dimension of the estimation problem, thereby achieving greater estimation precision than is possible with fully nonparametric methods, but provide greater flexibility in the shape of the regression function than is possible with parametric models. This makes additive modeling attractive when simple parametric models do not fit the data well. Other examples of dimension reduction methods are index models (e.g., Ichimura (1993), Powell, 
Stock, and Stoker (1989), and Hristache, Juditsky, and Spokoiny (2001) for mean regression models; Chaudhuri, Doksum, and Samarov (1997) and Khan (2001) for quantile regression models) and partially linear models (e.g., Robinson (1988) for mean regression models; He and Shi (1996) and Lee (2003) for quantile regression models). These are non-nested with additive models and, therefore, not substitutes for them. Examples of empirical applications of nonparametric additive models include: Hastie and Tibshirani (1990), Fan and Gijbels (1996), and Horowitz and Lee (2002), among many others.

To the best of our knowledge, there are three existing methods for estimating model (1.1): spline, backfitting, and marginal integration estimators. Doksom and Koo (2000) consider a spline estimator, but they do not provide pointwise rates of convergence or an asymptotic distribution. This makes inference with spline estimators difficult, though it is not necessarily impossible if the sample is sufficiently large. Bertail, Politis, and Romano (1999) show how to use subsampling to carry out inference when neither the asymptotic distribution nor the pointwise rate of convergence of an estimator is known. It is not known whether spline estimators can achieve the optimal pointwise rate. Huang (2003) discusses the difficulty of obtaining pointwise asymptotic normality with spline estimators in the context of additive nonparametric mean regression models. Fan and Gijbels (1996, pp. 296297) propose a backfitting estimator of (1.1). However, as with spline estimators, the rate of convergence and other asymptotic distributional properties of the backfitting estimator are unknown. De Gooijer and Zerom (2003) develop a marginal integration estimator of (1.1). This estimator is asymptotically normal and, therefore, enables inference to be carried out in a relatively straightforward way. However, marginal integration begins with an unrestricted, $d$-dimensional, nonparametric quantile regression. Consequently, marginal integration suffers from the curse of dimensionality and is likely to be imprecise when $d$ is large (see Remark 6 of De Gooijer and Zerom 2003). In summary, existing methods for estimating (1.1) either have unknown pointwise rates of convergence and asymptotic distributions, which makes inference difficult, or suffer from the curse of dimensionality, which makes them imprecise in multidimensional settings.

This paper presents an estimator of (1.1) that is asymptotically normally distributed, thereby permitting relatively straightforward inference in applications, and avoids the curse of dimensionality. We show through theoretical calculations and Monte Carlo experiments that our estimator is more precise than the marginal integration estimator when $d$ is large. The comparison with the marginal integration estimator is important because marginal 
integration is the only other existing method that has a known rate of convergence and asymptotic distribution.

The estimator presented here builds on Horowitz and Mammen (2004) (hereinafter HM), who develop an estimator of the additive components of a nonparametric additive mean regression model with a link function. The estimator of HM converges in probability pointwise at the rate $n^{-2 / 5}$ when the additive components are twice continuously differentiable, regardless of the dimension of $\mathbf{X}$. Thus, the estimator has no curse of dimensionality. This paper extends the HM approach to additive quantile regression models. As in HM, we use a two-stage estimation procedure that does not require full-dimensional, unrestricted nonparametric estimation. In the first stage, the additive components are estimated by a series quantile-regression estimator that imposes the additive structure of (1.1). In the second stage, the estimator of each additive component is obtained by a one-dimensional local polynomial quantile regression in which the other components are replaced by their first-stage series estimates. Although the estimation method proposed here is similar in concept to that of HM, mean and quantile regressions are sufficiently different to make the extension of HM to quantile regressions non-trivial and to require a separate treatment.

The key to the ability of our estimator to avoid the curse of dimensionality is that by imposing additivity at the outset, the first-stage series estimator achieves a faster-converging bias than does a full-dimensional nonparametric estimator. Although the variance of the series estimator converges relatively slowly, the second estimation step creates an averaging effect that reduces variance, thereby achieving the optimal rate of convergence. The approach used here differs from typical two-stage estimation, which aims at estimating a single parameter by updating an initial consistent estimator. Here, there are several unknown functions, but we update the estimator of only one. We show that asymptotically, the estimation error of the other functions does not appear in the updated estimator of the function of interest.

The remainder of this paper is organized as follows. Section 2 provides an informal description of the two-stage estimator. Section 3 applies the estimator to an empirical example. Asymptotic properties of the estimator are given in Section 4. Section 5 reports the results of a Monte Carlo investigation in which we compare the finite sample performance of the new estimator and two existing estimators. Concluding comments are in Section 6 . All the proofs are in the Appendix. We use the following notation. We let subscripts index observations of random variables and superscripts denote components of vectors. Thus, if 
$\mathbf{X}$ is a random vector, $\mathbf{X}_{i}$ is the $i$ 'th observation of $\mathbf{X}, X^{j}$ is the $j^{\prime}$ th component of $\mathbf{X}$, and $X_{i}^{j}$ is the $i$ 'th observation of the $j$ 'th component. We suppress the subscript $\alpha$ in the notation whenever this can be done without causing confusion.

\section{Informal Description of the Estimator}

This section describes a two-stage procedure for estimating $m_{j}(\cdot)$. For any $\mathbf{x} \in \mathbf{R}^{d}$, define $m(\mathbf{x})=m_{1}\left(x^{1}\right)+\ldots+m_{d}\left(x^{d}\right)$, where $x^{j}$ is the $j$-th component of $\mathbf{x}$. We assume that the support of $\mathbf{X}$ is $\mathcal{X} \equiv[-1,1]^{d}$, and normalize $m_{1}, \ldots, m_{d}$ so that

$$
\int_{-1}^{1} m_{j}(v) d v=0
$$

for $j=1, \ldots, d$. There is no loss of generality of this normalization since the location of $m_{j}$ is not identified without further restrictions.

To describe the first-stage series estimator, let $\left\{p_{k}: k=1,2, \cdots\right\}$ denote a basis for smooth functions on $[-1,1]$. Conditions that the basis functions must satisfy are given in Section 4 . For any positive integer $\kappa$, define

$$
P_{\kappa}(\mathbf{x})=\left[1, p_{1}\left(x^{1}\right), \ldots, p_{\kappa}\left(x^{1}\right), p_{1}\left(x^{2}\right), \ldots, p_{\kappa}\left(x^{2}\right), \ldots, p_{1}\left(x^{d}\right), \ldots, p_{\kappa}\left(x^{d}\right)\right]^{\prime}
$$

Then for $\theta_{\kappa} \in \mathbf{R}^{\kappa d+1}, P_{\kappa}(\mathbf{x})^{\prime} \theta_{\kappa}$ is a series approximation to $\mu+m(\mathbf{x})$. To obtain asymptotic results, $\kappa$ must satisfy certain conditions as $n \rightarrow \infty$. Upper and lower bounds on the number of terms $\kappa$ are given in Section 4 . For a random sample $\left\{\left(Y_{i}, \mathbf{X}_{i}\right): i=1, \ldots, n\right\}$, let $\hat{\theta}_{n \kappa}$ be a solution to

$$
\min _{\theta} S_{n \kappa}(\theta) \equiv n^{-1} \sum_{i=1}^{n} \rho_{\alpha}\left[Y_{i}-P_{\kappa}\left(\mathbf{X}_{i}\right)^{\prime} \theta\right]
$$

where $\rho_{\alpha}(u)=|u|+(2 \alpha-1) u$ for $0<\alpha<1$ is the the check function. The first-stage series estimator of $\mu+m(\mathbf{x})$ is defined as

$$
\tilde{\mu}+\tilde{m}(\mathbf{x})=P_{\kappa}(\mathbf{x})^{\prime} \hat{\theta}_{n \kappa}
$$

where $\tilde{\mu}$ is the first component of $\hat{\theta}_{n \kappa}$. For any $j=1, \ldots, d$ and any $x^{j} \in[-1,1]$, the series estimator $\tilde{m}_{j}\left(x^{j}\right)$ of $m_{j}\left(x^{j}\right)$ is the product of $\left[p_{1}\left(x^{j}\right), \cdots, p_{\kappa}\left(x^{j}\right)\right]$ with the appropriate components of $\hat{\theta}_{n \kappa}$. The same basis functions $\left\{p_{1}, \ldots, p_{k}\right\}$ are used to approximate each $m_{j}(\cdot)$. No cross products are needed because of the additive form of (1.1). 
To describe the second-stage estimator of (say) $m_{1}\left(x^{1}\right)$, define

$$
m_{-1}\left(\tilde{\mathbf{X}}_{i}\right)=m_{2}\left(X_{i}^{2}\right)+\cdots+m_{d}\left(X_{i}^{d}\right) \text { and } \tilde{m}_{-1}\left(\tilde{\mathbf{X}}_{i}\right)=\tilde{m}_{2}\left(X_{i}^{2}\right)+\cdots+\tilde{m}_{d}\left(X_{i}^{d}\right),
$$

where $\tilde{\mathbf{X}}_{i}=\left(X_{i}^{2}, \ldots, X_{i}^{d}\right)$. Assume that $m_{1}$ is at least $r$-times continuously differentiable on $[-1,1]$. Then the second-stage estimator of $m_{1}\left(x^{1}\right)$ is a $(r-1)$-th local polynomial estimator with $m_{-1}\left(\tilde{\mathbf{X}}_{i}\right)$ replaced by the first-stage estimates $\tilde{m}_{-1}\left(\tilde{\mathbf{X}}_{i}\right)$. Specifically, the estimator $\hat{m}_{1}\left(x^{1}\right)$ of $m_{1}\left(x^{1}\right)$ is defined as $\hat{m}_{1}\left(x^{1}\right)=\hat{b}_{0}$, where $\hat{\mathbf{b}}_{n}=\left(\hat{b}_{0}, \hat{b}_{1}, \ldots, \hat{b}_{r-1}\right)$ minimizes

$$
S_{n}(b) \equiv\left(n \delta_{n}\right)^{-1} \sum_{i=1}^{n} \rho_{\alpha}\left[Y_{i}-\tilde{\mu}-b_{0}-\sum_{k=1}^{r-1} b_{k}\left[\delta_{n}^{-1}\left(X_{i}^{1}-x^{1}\right)\right]^{k}-\tilde{m}_{-1}\left(\tilde{\mathbf{X}}_{i}\right)\right] K\left(\frac{x^{1}-X_{i}^{1}}{\delta_{n}}\right),
$$

$K$ (kernel function) is a probability density function on $[-1,1]$, and $\delta_{n}$ (bandwidth) is a sequence of real numbers converging to zero as $n \rightarrow \infty$. The regularity conditions for $K$ and $\delta_{n}$ are given in Section 4. The second-stage estimators of $m_{2}\left(x^{2}\right), \ldots, m_{d}\left(x^{d}\right)$ are obtained similarly. The estimator of the regression surface is $\tilde{\mu}+\hat{m}_{1}\left(x^{1}\right)+\ldots+\hat{m}_{d}\left(x^{d}\right)$. The value of $r$ in (2.2) can vary according to the additive component $m_{j}$ that is being estimated if different components are known to have different orders of differentiability. If the orders of differentiability of the $m_{j}$ 's are unknown in an application, we suggest setting $r=2$ (local linear estimation). This achieves dimension reduction and reasonable precision without assuming the existence of higher-order derivatives.

Since quantile regression is equivariant to monotone transformations of $Y$ (i.e., quantiles of monotone transformations of $Y$ are equal to monotone transformations of quantiles of $Y)$, it is straightforward to extend the estimator of (1.1) to a generalized additive model that has the form

$$
G(Y)=\mu+m_{1}\left(X^{1}\right)+\cdots+m_{d}\left(X^{d}\right)+U
$$

where $G$ is a known, strictly increasing function, and the $\alpha$-th quantile of $U$ conditional $\mathbf{X}=\mathbf{x}$ is zero for almost every $\mathbf{x}$. The estimator of the $\alpha$-th quantile of $Y$ conditional $\mathbf{X}=\mathbf{x}$ can be easily obtained by $G^{-1}\left[\tilde{\mu}+\hat{m}_{1}\left(x^{1}\right)+\cdots+\hat{m}_{d}\left(x^{d}\right)\right]$, where $\tilde{\mu}+\hat{m}_{1}\left(x^{1}\right)+\cdots+\hat{m}_{d}\left(x^{d}\right)$ is obtained by the estimation procedure described above with $G\left(Y_{i}\right)$ being substituted for $Y_{i}$.

We end this section by mentioning computational aspects of the estimation procedure. Both the first-stage and second-stage minimization problems, (2.1) and (2.2) are linear programming problems and therefore can be solved easily by using computation methods 
developed for linear quantile regression methods. Moreover, the new estimator does not require iterations (backfitting approach) or $n$ first-stage estimates (marginal integration method).

\section{An Empirical Example}

This section provides an empirical example that illustrates the usefulness of our estimator. Yafeh and Yosha (2003) used a sample of Japanese firms in the chemical industry to examine whether 'concentrated shareholding is associated with lower expenditure on activities with scope for managerial private benefits'. In this section, we concentrate on only one of regressions considered by Yafeh and Yosha (2003). The dependent variable $Y$ is general sales and administrative expenses deflated by sales (denoted by MH5 in Yafeh and Yosha (2003)). This measure is one of five measures of expenditures on activities with scope for managerial private benefits considered by Yafeh and Yosha (2003). The covariates include a measure of ownership concentration (denoted by TOPTEN, cumulative shareholding by the largest ten shareholders), and firm characteristics: the log of assets, firm age, and leverage (the ratio of debt to debt plus equity). The regression model used by Yafeh and Yosha (2003) is

$$
\mathrm{MH} 5=\beta_{0}+\beta_{1} \text { TOPTEN }+\beta_{2} \log (\text { Assets })+\beta_{3} \text { Age }+\beta_{4} \text { Leverage }+U
$$

The sample size is 185 . This dataset is available at the Economic Journal web site at http://www.res.org.uk.

We estimated the additive conditional median function using the two-stage estimator. Estimation results for other conditional quantile functions are available on request. Before estimation begins, the covariates are standardized to have mean zero and variance $1 . B$ splines were used for the first-stage with $\kappa_{n}=4$, which is equal to $\hat{\kappa}_{n}+1$, where $\hat{\kappa}_{n}=3$ minimizes the following Schwarz-type information criterion (see He and Shi (1996) and Doksum and Koo (2000))

$$
Q B I C\left(\kappa_{n}\right)=n \log \left(\sum_{i=1}^{n} \rho_{\alpha}\left[Y_{i}-P_{\kappa}\left(\mathbf{X}_{i}\right)^{\prime} \hat{\theta}_{n \kappa}\right]\right)+2(\log n) \kappa_{n} .
$$

Overfitting is needed to reduce the asymptotic bias (see Assumption 4.8 (a)). Local linear fitting was used for the second-stage with the bandwidths $\delta_{n}=(0.42,0.40,0.45,0.47)$ for estimation of each additive component, respectively. The bandwidths $\delta_{n}$ are chosen by 
using a simple rule of thumb described in Fan and Gijbels (1996, p.202). The kernel $K$ is taken to be the normal density function. Varying $\delta_{n}$ from $0.75 \delta_{n}$ to $1.25 \delta_{n}$ did not change estimation results significantly. All the computation in this paper is carried out in R using libraries 'splines' (to generate $B$-spline basis) and 'quantreg' (to solve (2.1) and (2.2)). The $\mathrm{R}$ language is available as free software at http://www.r-project.org.

Figure 1 summarizes estimation results. Each panel shows the estimated function of interest and $90 \%$ symmetric pointwise confidence interval (without bias correction). The confidence interval was obtained using asymptotic approximation based on Theorem 4.3. The unknown components of the asymptotic variance in Theorem 4.3 were estimated by kernel density estimators.

It can be seen that the effects of ownership concentration (TOPTEN) are nonlinear. This suggests that the relationship between MH5 and TOPTEN conditional on firm characteristics cannot be well described by a linear location-shift model. The effects of firm size $(\log ($ Assets $))$ are also highly nonlinear. This may be due to the fact that MH5 includes expenditures that are not related with managerial private benefits. The effects of firm age are also very nonlinear, indicating that newly established firms are different from mature firms. The effects of leverage are quite linear.

The estimation results suggest that the linear model is misspecified. To verify this, we used the test of linearity for median regression models in Horowitz and Spokoiny (2002). The test gives a test statistic of 2.192 with a $5 \%$ critical value of 1.999 . Thus, the test rejects the linear median regression model at the $5 \%$ level. Rejection of linearity does not mean necessarily that the additive model fits the data well. To check whether the additive model is a good fit, we consider an informal graphical method. Figure 2 shows plots of estimated residuals against covariates. It can be seen that residuals scatter around and show no obvious evidence of misspecification, although the plots show some skewness. To check additivity formally would require a specification test. None is available in the literature, and developing one is beyond the scope of this paper. Tests for additivity of a mean regression model are available (see, for example, Gozalo and Linton (2001)), but these have not been extended to quantile regressions.

In summary, our estimation results indicate that a model that is more flexible than a linear median regression model is needed to study the relationship between concentrated shareholding and expenditures for managerial private benefits. 


\section{Asymptotic Results}

This section gives asymptotic results for the estimator described in Section 2. We need some additional notation. For any matrix $A$, let $\|A\|=\left[\operatorname{trace}\left(A^{\prime} A\right)\right]^{1 / 2}$ be the Euclidean norm. Let $d(\kappa)=\kappa d+1$ and $b_{\kappa 0}(\mathbf{x})=\mu+m(\mathbf{x})-P_{\kappa}(\mathbf{x})^{\prime} \theta_{\kappa 0}$.

To establish asymptotic results, we need the following conditions.

Assumption 4.1. The data $\left\{\left(Y_{i}, \mathbf{X}_{i}\right): i=1, \ldots, n\right\}$ are i.i.d. and the $\alpha$-th quantile of $Y$ conditional on $\mathbf{X}=\mathbf{x}$ is $\mu+m(\mathbf{x})$ for almost every $\mathbf{x} \in \mathcal{X}$.

Assumption 4.2. The support of $\mathbf{X}$ is $\mathcal{X} \equiv[-1,1]^{d}$. The distribution of $\mathbf{X}$ is absolutely continuous with respect to Lebesgue measure. The probability density function of $\mathbf{X}$ (denoted by $f_{\mathbf{X}}(\mathbf{x})$ ) is bounded, is bounded away from zero, is twice continuously differentiable in the interior of $\mathcal{X}$, and has continuous second-order one-sided derivatives at the boundary of $\mathcal{X}$.

Assumption 4.3. Let $F(u \mid \mathbf{x})$ be the distribution function of $U_{\alpha}$ conditional on $\mathbf{X}=\mathbf{x}$. Assume that $F(0 \mid \mathbf{x})=\alpha$ for almost every $\mathbf{x} \in \mathcal{X}$ and that $F(\cdot \mid \mathbf{x})$ has a probability density function $f(\cdot \mid \mathbf{x})$. There is a constant $L_{f}<\infty$ such that $\left|f\left(u_{1} \mid \mathbf{x}\right)-f\left(u_{2} \mid \mathbf{x}\right)\right| \leq L_{f}\left|u_{1}-u_{2}\right|$ for all $u_{1}$ and $u_{2}$ in a neighborhood of zero and for all $\mathbf{x} \in \mathcal{X}$. Also, there are constants $c_{f}>0$ and $C_{f}<\infty$ such that $c_{f} \leq f(u \mid \mathbf{x}) \leq C_{f}$ for all $u$ in a neighborhood of zero and for all $\mathbf{x} \in \mathcal{X}$.

Assumption 4.4. For each $j, m_{j}(\cdot)$ is r-times continuously differentiable in the interior of $[-1,1]$ and has continuous $r$-th-order one-sided derivatives at the boundary of $[-1,1]$ for some $r \geq 2$.

Assumption 4.5. Define $\Phi_{\kappa}=E\left[f(0 \mid \mathbf{X}) P_{\kappa}(\mathbf{X}) P_{\kappa}(\mathbf{X})^{\prime}\right]$. The smallest eigenvalue of $\Phi_{\kappa}$ is bounded away from zero for all $\kappa$, and the largest eigenvalue of $\Phi_{\kappa}$ is bounded for all $\kappa$.

Assumption 4.6. Define $\zeta_{\kappa}=\sup _{\mathbf{x} \in \mathcal{X}}\left\|P_{\kappa}(\mathbf{x})\right\|$. The basis functions $\left\{p_{k}: k=1,2, \ldots\right\}$ satisfy the following conditions:

(a) each $p_{k}$ is continuous,

(b) $\int_{-1}^{1} p_{k}(v) d v=0$,

$$
\text { (c) } \int_{-1}^{1} p_{j}(v) p_{k}(v) d v=\left\{\begin{array}{cc}
1 & \text { if } j=k \\
0 & \text { otherwise }
\end{array}\right.
$$


(d) as $\kappa \rightarrow \infty$,

$$
\zeta_{\kappa}=O\left(\kappa^{1 / 2}\right)
$$

(e) there are vectors $\theta_{\kappa 0} \in \mathbf{R}^{d(\kappa)}$ such that

$$
\sup _{\mathbf{x} \in \mathcal{X}}\left|\mu+m(\mathbf{x})-P_{\kappa}(\mathbf{x})^{\prime} \theta_{\kappa 0}\right|=O\left(\kappa^{-r}\right)
$$

as $\kappa \rightarrow \infty$.

Assumption 4.7. $\left(\kappa^{4} / n\right)(\log n)^{2} \rightarrow 0$ and $\kappa^{1+2 r} / n$ is bounded.

Assumption 4.1 defines a data generating process. If necessary, the bounded support condition in Assumption 4.2 can be satisfied by carrying out a monotone transformation of $\mathbf{X}$, provided that the probability density function of the transformed $\mathbf{X}$ is bounded away from zero. Among other things, Assumption 4.3 requires that $f(\cdot \mid \mathbf{x})$ be bounded away from zero in a neighborhood of zero uniformly over $\mathbf{x}$. This is a convenient condition to establish asymptotic results. Without this condition, the rate of convergence could be slower and the asymptotic distribution could be non-normal. See, for example, Knight (1998) for asymptotic results for linear median regression estimators under more general assumptions on $f(u \mid \mathbf{x})$. The smoothness assumptions on $F(u \mid \mathbf{x})$ in Assumption 4.3 and on $m_{j}(\cdot)$ in Assumption 4.4 are reasonable in a wide variety of applications and include most parametric specifications of $F(u \mid \mathbf{x})$ and $m_{j}(\cdot)$ as special cases. As in Newey (1997) and HM, Assumption 4.5 insures the non-singularity of the covariance matrix of the asymptotic form of the first-stage estimator. Assumption 4.6 imposes restrictions on the basis functions. When $f_{\mathbf{X}}$ is bounded away from zero and $m_{j}$ is continuously $r$-times differentiable, the conditions in Assumption 4.6 are satisfied by B-splines. Assumption 4.6 (e) restricts the order of asymptotic bias. Due to the additive structure of (1.1), the uniform approximation error in (4.2) is of order $O\left(\kappa^{-r}\right)$ regardless of the dimension of $\mathbf{X}$. This helps the secondstage estimator avoid the curse of dimensionality.

The following theorem gives a uniform convergence result for the first-stage series estimator.

Theorem 4.1. Let Assumptions 4.1-4.7 hold. Then as $n \rightarrow \infty$,

$$
\text { (a) }\left\|\hat{\theta}_{n \kappa}-\theta_{\kappa 0}\right\|=O_{p}\left[(\kappa / n)^{1 / 2}\right],
$$


and

$$
\text { (b) } \sup _{\mathbf{x} \in \mathcal{X}}|\tilde{m}(\mathbf{x})-m(\mathbf{x})|=O_{p}\left[\kappa /\left(n^{1 / 2}\right)\right] .
$$

He and Shi $(1994,1996)$ obtained $L_{2}$ rates of convergence for B-spline estimators of univariate and bivariate quantile regression models and Portnoy (1997) and He and Portnoy (2000) derived local asymptotic properties of smoothing splines for $d \leq 2$.

The following theorem establishes a Bahadur-type expansion of the first-stage estimator.

Theorem 4.2. Let Assumptions 4.1-4.7 hold. Then as $n \rightarrow \infty$,

$\hat{\theta}_{n \kappa}-\theta_{\kappa 0}=n^{-1} \Phi_{\kappa}^{-1} \sum_{i=1}^{n} P_{\kappa}\left(\mathbf{X}_{i}\right)\left\{\alpha-1\left[U_{i} \leq 0\right]\right\}+n^{-1} \Phi_{\kappa}^{-1} \sum_{i=1}^{n} f\left(0 \mid \mathbf{X}_{i}\right) P_{\kappa}\left(\mathbf{X}_{i}\right) b_{\kappa 0}\left(\mathbf{X}_{i}\right)+R_{n}$

where the remainder term $R_{n}$ satisfies

$$
\left\|R_{n}\right\|=O_{p}\left[\left(\kappa^{2} / n\right)^{3 / 4}(\log n)^{1 / 2}+\kappa^{3 / 2} / n\right]+o_{p}\left(n^{-1 / 2}\right)
$$

To state asymptotic results for the second-stage estimator, we need additional assumptions.

Assumption 4.8. (a) $\kappa=C_{\kappa} n^{\nu}$ for some constant $C_{\kappa}$ satisfying $0<C_{\kappa}<\infty$ and some $\nu$ satisfying

$$
\frac{1}{2 r+1}<\nu<\frac{2 r+3}{12 r+6}
$$

(b) $\delta_{n}=C_{\delta} n^{-1 /(2 r+1)}$ for some constant $C_{\delta}$ satisfying $0<C_{\delta}<\infty$.

Assumption 4.9. The function $K$ is a bounded, continuous probability density function on $[-1,1]$ and is symmetrical about 0 .

Assumption 4.8 (a) requires that $r \geq 2$. Assumption 4.8 (b) and Assumption 4.9 are standard in the nonparametric estimation literature.

Define

$$
\bar{P}_{\kappa}(\tilde{\mathbf{x}})=[1, \underbrace{0, \ldots, 0}_{\kappa}, p_{1}\left(x^{2}\right), \ldots, p_{\kappa}\left(x^{2}\right), \ldots, p_{1}\left(x^{d}\right), \ldots, p_{\kappa}\left(x^{d}\right)]^{\prime}
$$

where $\tilde{\mathbf{x}}=\left(x^{2}, \ldots, x^{d}\right)$. The following condition is used to establish the limiting distribution of the two-stage estimator. 
Assumption 4.10. The largest eigenvalue of $E\left[\bar{P}_{\kappa}(\tilde{\mathbf{X}}) \bar{P}_{\kappa}(\tilde{\mathbf{X}})^{\prime} \mid X^{1}=x^{1}\right]$ is bounded for all $\kappa$ and each component of $E\left[\bar{P}_{\kappa}(\tilde{\mathbf{X}}) \bar{P}_{\kappa}(\tilde{\mathbf{X}})^{\prime} \mid X^{1}=x^{1}\right]$ is twice continuously differentiable with respect to $x^{1}$.

Let $\mu_{j}=\int_{-1}^{1} u^{j} K(u) d u$ denote the moments of $K$ and let $S(K)$ be the $(r \times r)$ matrix, whose $(i, j)$ component is $\mu_{i+j-2}$. Also, let $e_{1}=(1,0, \ldots, 0)$ be the unit column vector. As in Ruppert and Wand (1994) and Fan and Gijbels (1996, pp. 63-66), let $K_{*}(u)=$ $e_{1}^{\prime} S(K)^{-1}\left(1, u, \ldots, u^{r-1}\right)^{\prime} K(u)$ be the 'equivalent kernel'. $K_{*}$ is a kernel of order $r$ if $r$ is even and of order $r+1$ for odd $r$. The higher-order property of the equivalent kernel is useful for analyzing higher-order local polynomial fitting. Let $f_{X^{1}}\left(x^{1}\right)$ denote the probability density function of $X^{1}, f_{1}\left(u \mid x^{1}\right)$ the probability density function of $U_{\alpha}$ conditional on $X^{1}=x^{1}$, and $D^{k} m_{j}\left(x^{j}\right)$ the $k$-th order derivative of $m_{j}$. The main result of the paper is as follows:

Theorem 4.3. Let Assumptions 4.1-4.10 hold. Also, assume that $r \geq 2$ is even, where $r$ is defined in Assumption 4.4. Then as $n \rightarrow \infty$, for any $x^{1}$ satisfying $\left|x^{1}\right| \leq 1-\delta_{n}$,

$$
\begin{aligned}
& \text { (a) }\left|\hat{m}_{1}\left(x^{1}\right)-m_{1}\left(x^{1}\right)\right|=O_{p}\left[n^{-r /(2 r+1)}\right] . \\
& \text { (b) } n^{r /(2 r+1)}\left[\hat{m}_{1}\left(x^{1}\right)-m_{1}\left(x^{1}\right)\right] \rightarrow_{d} \mathbf{N}\left[B\left(x^{1}\right), V\left(x^{1}\right)\right], \\
& \text { where } \\
& B\left(x^{1}\right)=\left\{\int_{-1}^{1} u^{r} K_{*}(u) d u\right\}\{r !\}^{-1} C_{\delta}^{r} D^{r} m_{1}\left(x^{1}\right) \text { and } \\
& V\left(x^{1}\right)=\left\{\int_{-1}^{1}\left[K_{*}(u)\right]^{2} d u\right\} C_{\delta}^{-1} \alpha(1-\alpha) /\left\{f_{X^{1}}\left(x^{1}\right)\left[f_{1}\left(0 \mid x^{1}\right)\right]^{2}\right\} .
\end{aligned}
$$

(c) If $j \neq 1$, then $n^{r /(2 r+1)}\left[\hat{m}_{1}\left(x^{1}\right)-m_{1}\left(x^{1}\right)\right]$ and $n^{r /(2 r+1)}\left[\hat{m}_{j}\left(x^{j}\right)-m_{j}\left(x^{j}\right)\right]$ are asymptotically independently normally distributed for any $x^{j}$ satisfying $\left|x^{j}\right| \leq 1-\delta_{n}$.

The theorem implies that the second-stage estimator achieves the optimal rate of convergence for a nonparametric estimator of a function with $r$ derivatives. In addition, it has the same asymptotic distribution that it would have if $m_{2}, \ldots, m_{d}$ were known. Parts (b) and (c) of Theorem 4.3 imply that estimators of $m_{1}\left(x^{1}\right), \ldots, m_{d}\left(x^{d}\right)$ are asymptotically independently distributed. Because of Assumption 4.8 (a), it is required that $m_{j}$ be at least twice continuously differentiable. This required differentiability is independent of the dimension of $\mathbf{X}$ and, therefore, our estimator avoids the curse of dimensionality. Although Theorem 4.3 is established only for interior points of $\mathcal{X}$, it is expected that the second-stage estimator does not require boundary modifications (see Fan, Hu, and Truong (1994) for asymptotic properties of the local linear quantile regression estimator at boundary points). 
When $(r-2)$ local polynomial fits are used in (2.2) with even $r \geq 2$ (for example, a local constant estimator is used under the assumption that $r=2$ ), the results in the theorem would be the same except that the asymptotic bias depends on $D f_{X^{1}}\left(x^{1}\right) / f_{X^{1}}\left(x^{1}\right)$ and therefore is not design-adaptive. To be specific, in this case $B\left(x^{1}\right)$ in Theorem 4.3 has the form

$$
B\left(x^{1}\right)=\left\{\int_{-1}^{1} u^{r} K_{*}(u) d u\right\}\left\{\frac{D^{r-1} m_{1}\left(x^{1}\right) D f_{X^{1}}\left(x^{1}\right)}{(r-1) ! f_{X^{1}}\left(x^{1}\right)}+\frac{D^{r} m_{1}\left(x^{1}\right)}{r !}\right\} C_{\delta}^{r} .
$$

When $r \geq 2$ is odd, for any $x^{1}$ satisfying $\left|x^{1}\right| \leq 1-\delta_{n}$, it can be shown that

$$
n^{(r+1) /(2 r+3)}\left[\hat{m}_{1}\left(x^{1}\right)-m_{1}\left(x^{1}\right)\right] \rightarrow_{d} \mathbf{N}\left[B\left(x^{1}\right), V\left(x^{1}\right)\right],
$$

where

$$
\begin{aligned}
& B\left(x^{1}\right)=\left\{\int_{-1}^{1} u^{r+1} K_{*}(u) d u\right\}\left\{\frac{D^{r} m_{1}\left(x^{1}\right) D f_{X^{1}}\left(x^{1}\right)}{r ! f_{X^{1}}\left(x^{1}\right)}+\frac{D^{r+1} m_{1}\left(x^{1}\right)}{(r+1) !}\right\} C_{\delta}^{r+1} \text { and } \\
& V\left(x^{1}\right)=\left\{\int_{-1}^{1}\left[K_{*}(u)\right]^{2} d u\right\} C_{\delta}^{-1} \alpha(1-\alpha) /\left\{f_{X^{1}}\left(x^{1}\right)\left[f_{1}\left(0 \mid x^{1}\right)\right]^{2}\right\}
\end{aligned}
$$

with $\delta_{n}=C_{\delta} n^{-1 /(2 r+3)}$ for some constant $C_{\delta}$ satisfying $0<C_{\delta}<\infty$. Again, the asymptotic variance is the same but the asymptotic bias involves $D f_{X^{1}}\left(x^{1}\right) / f_{X^{1}}\left(x^{1}\right)$. See, for example, Ruppert and Wand (1994) and Fan and Gijbels (1996, pp. 61-63) for the asymptotic bias and variance of estimators of local polynomial mean regression models.

\section{Monte Carlo Experiments}

This section reports the results of a small set of Monte Carlo experiments in which we compare the finite-sample performance of the two-stage estimator and two existing estimators. We first compare the performance of the two-stage estimator with that of the marginal integration estimator of De Gooijer and Zerom (2003). Experiments were carried out with $d=2$ and $d=5$. The experiments with $d=2$ were carried out with the design identical to that of De Gooijer and Zerom (2003, Section 4). Specifically, the experiments consist of estimating $m_{1}$ and $m_{2}$ in

$$
Y=m_{1}\left(X^{1}\right)+m_{2}\left(X^{2}\right)+0.25 \varepsilon
$$

where $m_{1}\left(x^{1}\right)=0.75 x^{1}$ and $m_{2}\left(x^{2}\right)=1.5 \sin \left(0.5 \pi x^{2}\right)$. The covariates $X^{1}$ and $X^{2}$ are bivariate normal with mean zero, unit variance, and correlation $\rho$. We consider $\alpha=0.5$ 
and sample sizes $n=100$ and 200. As in De Gooijer and Zerom (2003), experiments were carried out with $\rho=0.2$ (low correlation between covariates) and $\rho=0.8$ (high correlation).

$B$-splines were used for the first-stage of the two-stage estimator with $\kappa_{n}=4$ and local linear fitting was used for the second-stage. Also, the kernel $K$ is taken to be the normal density function. The bandwidth $\delta_{1 n}=3 \hat{\sigma}_{X^{1}} n^{-1 / 5}$ was chosen for estimation of $m_{1}$ and $\delta_{2 n}=\hat{\sigma}_{X^{2}} n^{-1 / 5}$ was for estimation of $m_{2}$, where $\hat{\sigma}_{X^{j}}$ is the sample standard deviation of $X^{j}$ for $j=1,2$. The normal density function does not satisfy the finite support condition in Assumption 4.9, but these kernel and bandwidths were chosen to be identical to those in De Gooijer and Zerom (2003) in order to compare the finite-sample performance of the two-stage estimator vis-à-vis those of the marginal integration method and the backfitting approach reported in De Gooijer and Zerom (2003).

To see whether the two-stage estimator avoids the curse of dimensionality in finitesamples, three additional covariates were added to (5.1). More specifically, the experiments with $d=5$ consist of estimating $m_{1}$ and $m_{2}$ in

$$
Y=m_{1}\left(X^{1}\right)+m_{2}\left(X^{2}\right)+X^{3}+X^{4}+X^{5}+0.25 \varepsilon
$$

where $X^{j}$ are independently distributed as $U[-1,1]$ for $j=3,4,5$. Since the local linear fitting is used, the second stage estimator has the rate of convergence $n^{-2 / 5}$ regardless of $d$.

There were 100 Monte Carlo replications per experiment and the absolute deviation error (ADE) was computed for each estimated function on the interval $[-2,2]$. An average of the ADE's (AADE) was the criterion used in De Gooijer and Zerom (2003).

The upper panel of Table 1 shows the AADE values for the marginal integration and two-stage estimators for combinations of $d, \rho$ and $n$. We computed the pilot estimator of the marginal integration estimator directly through the check function method, whereas De Gooijer and Zerom (2003) obtained the pilot estimator by inverting the conditional distribution function. As in De Gooijer and Zerom (2003), local linear approximation is adopted in the direction of interest and local constant approximation is used in the nuisance directions. The asymptotic distribution of the marginal integration estimator is identical regardless of the choice between two alternative pilot estimators. As was reported by De Gooijer and Zerom (2003), the marginal integration estimator performs poorly when there is high correlation among covariates. When $d=2$ and $\rho=0.8$, the finite-sample performance of the two-stage estimator is considerably better than that of the mariginal integration estimator. Also, the performance of the two-stage estimator is comparable between $\rho=0.2$ 
and $\rho=0.8$ when $d=2$. That is also the case between $d=2$ and $d=5$ for both $\rho=0.2$ and $\rho=0.8$. These are consistent with the asymptotic results established in Section 4 because the limiting distribution of the two-stage estimator does not depend on $d$ or $\rho$. However, the marginal integration estimator performs very poorly when $d=5$ and $\rho=0.8$. In that case, the AADE's for the marginal integration estimator are more than twice as large as those for the two-stage estimator.

We now compare the finite-sample performance of the two-stage estimator with a spline estimator. We consider the same Monte Carlo design with $d=2$. $B$-splines were used for the spline estimator with data-dependent choice of $\hat{\kappa}_{n}$. Specifically, $\hat{\kappa}_{n}$ is chosen by minimizing Schwarz-type information criterion given by (3.2). For the first-stage of the two-stage estimator, $\hat{\kappa}_{n}+1$ is used since asymptotic results require overfitting. For the second-stage of the two-stage estimator, the bandwidth $\delta_{n}$ is chosen by using a simple rule of thumb described in Fan and Gijbels (1996, p.202). This time there were 1000 Monte Carlo replications per experiment since the spline and two-stage estimators do not entail long computation times. The lower panel of Table 1 shows the AADE values for the spline and two-stage estimators for combinations of $\rho$ and $n$. Notice that the finite-sample performance of the two-stage estimator is marginally better for estimation of $m_{1}$ and virtually as good as that of the spline estimator for estimation of $m_{2}$. One could use an updated (second-stage) estimator of $m_{1}$ to estimate $m_{2}$ or vice versa. We carried out a Monte Carlo experiment for this approach, only finding that there was no gain in the finite sample performance for this additional updating (full results are not reported but available on request).

In summary, the results of experiments suggest that the two-stage estimator outperforms the marginal integration estimator when there is high correlation among covariates and/or the dimension of covariates is relatively large. Also, the experiment results indicate that the two-stage estimator performs better or as well as the spline estimator, with which it would be difficult to carry out inference.

\section{Conclusions}

This paper has developed an estimator of the additive components of a nonparametric additive quantile regression model. It is shown that the estimator converges in probability of $n^{-r /(2 r+1)}$ when the unknown functions are $r$-times continuously differentiable for some $r \geq 2$. This result holds regardless of the dimension of the covariates. In addition, the estimator has an oracle property. Specifically, the estimator of each additive component 
has the same asymptotic distribution that it would have if the other components were known. Finally, the estimator described here is easily extended to a generalized additive quantile regression model with a known link function. 


\section{A Appendix: Proofs}

Throughout the Appendix, let $C$ denote a generic constant that may be different in different uses. Let $\lambda_{\min }(A)$ and $\lambda_{\max }(A)$ denote minimum and maximum eigenvalues of a symmetric matrix $A$.

\section{A.1 Proofs of Theorems 4.1 and 4.2}

It is useful to introduce some additional notation that is used in Koenker and Bassett (1978) and Chaudhuri (1991). Let $\mathcal{N}=\{1, \ldots, n\}$ and $\mathcal{H}_{\kappa}$ denote the collection of all $d(\kappa)$-element subsets of $\mathcal{N}$. Also, let $B(h)$ denote the submatrix (subvector) of a matrix (vector) $B$ with rows (components) that are indexed by the elements of $h \in \mathcal{H}_{\kappa}$. In particular, let $\mathbf{P}_{\kappa}(h)$ denote a $d(\kappa) \times d(\kappa)$ matrix, whose rows are the vectors $P_{\kappa}\left(\mathbf{X}_{i}\right)^{\prime}$ such that $i \in h$, and let $\mathbf{Y}_{\kappa}(h)$ denote a $d(\kappa) \times 1$ vector, whose elements are $Y_{i}$ such that $i \in h$. Let $\mathbf{P}_{\kappa}$ denote a $n \times d(\kappa)$ matrix, whose rows are the vectors $P_{\kappa}\left(\mathbf{X}_{i}\right)^{\prime}$ for $i=1, \ldots, n$.

The following lemmas are useful to prove Theorems 4.1 and 4.2. The first lemma is from Koenker and Bassett (1978, Theorem 3.1).

Lemma A.1. Suppose that $\mathbf{P}_{\kappa}$ has rank $=d(\kappa)$. Then there is a subset $h_{\kappa} \in \mathcal{H}_{\kappa}$ such that the problem (2.1) has at least one solution of the form $\hat{\theta}_{n \kappa}=\mathbf{P}_{\kappa}\left(h_{\kappa}\right)^{-1} \mathbf{Y}_{\kappa}\left(h_{\kappa}\right)$.

Define

$$
\bar{H}_{n 1 \kappa}\left(\hat{\theta}_{n \kappa}\right)=\sum_{i=1, i \in h_{\kappa}^{c}}^{n}\left\{\alpha-1\left[Y_{i} \leq P_{\kappa}\left(\mathbf{X}_{i}\right)^{\prime} \hat{\theta}_{n \kappa}\right]\right\} P_{\kappa}\left(\mathbf{X}_{i}\right)^{\prime} \mathbf{P}_{\kappa}\left(h_{\kappa}\right)^{-1} .
$$

Lemma A.2. $\hat{\theta}_{n \kappa}=\mathbf{P}_{\kappa}\left(h_{\kappa}\right)^{-1} \mathbf{Y}_{\kappa}\left(h_{\kappa}\right)$ is a unique solution to (2.1) almost surely for all sufficiently large $n$.

Proof. The matrix $\mathbf{P}_{\kappa}$ has rank $=d(\kappa)$ almost surely for all sufficiently large $n$. By Lemma A.1, there exists a $h_{\kappa} \in \mathcal{H}_{\kappa}$ such that the problem (2.1) has at least one solution of the form $\hat{\theta}_{n \kappa}=$ $\mathbf{P}_{\kappa}\left(h_{\kappa}\right)^{-1} \mathbf{Y}_{\kappa}\left(h_{\kappa}\right)$.

As in Theorem 3.3 in Koenker and Bassett (1978) [see also Fact 6.4 in Chaudhuri (1991)], $\hat{\theta}_{n \kappa}=\mathbf{P}_{\kappa}\left(h_{\kappa}\right)^{-1} \mathbf{Y}_{\kappa}\left(h_{\kappa}\right)$ is a unique solution to $(2.1)$ if and only if each component in $\bar{H}_{n 1 \kappa}\left(\hat{\theta}_{n \kappa}\right)$ is strictly between $\alpha-1$ and $\alpha$, i.e. $\bar{H}_{n 1 \kappa}\left(\hat{\theta}_{n \kappa}\right) \in(\alpha-1, \alpha)^{d(\kappa)}$. Also, if $\hat{\theta}_{n \kappa}=\mathbf{P}_{\kappa}\left(h_{\kappa}\right)^{-1} \mathbf{Y}_{\kappa}\left(h_{\kappa}\right)$ is a solution to $(2.1)$, then $\bar{H}_{n 1 \kappa}\left(\hat{\theta}_{n \kappa}\right) \in[\alpha-1, \alpha]^{d(\kappa)}$.

Since the distribution of $P_{\kappa}\left(\mathbf{X}_{i}\right)$ is absolutely continuous with respect to Lebesgue measure (except for the first component), the probability that $\bar{H}_{n 1 \kappa}\left(\hat{\theta}_{n \kappa}\right)$ lies on the boundary of the cube $[\alpha-1, \alpha]^{d(\kappa)}$ is zero for all sufficiently large $n$. Therefore, $\hat{\theta}_{n \kappa}=\mathbf{P}_{\kappa}\left(h_{\kappa}\right)^{-1} \mathbf{Y}_{\kappa}\left(h_{\kappa}\right)$ is a unique solution almost surely for all sufficiently large $n$. 
Let $\Phi_{n \kappa}=n^{-1} \sum_{i=1}^{n} f\left(0 \mid \mathbf{X}_{i}\right) P_{\kappa}\left(\mathbf{X}_{i}\right) P_{\kappa}\left(\mathbf{X}_{i}\right)^{\prime}$. Let $1_{n}$ be the indicator function such that $1_{n}=$ $1\left\{\lambda_{\min }\left(\Phi_{n \kappa}\right) \geq \lambda_{\min }\left(\Phi_{\kappa}\right) / 2\right\}$. As in the proofs of Theorem 1 of Newey (1997) and Lemma 4 of HM, one can show that $\left\|\Phi_{n \kappa}-\Phi_{\kappa}\right\|^{2}=O_{p}\left(\kappa^{2} / n\right)=o_{p}(1)$. Thus, $\operatorname{Pr}\left(1_{n}=1\right) \rightarrow 1$ as $n \rightarrow \infty$.

Define

$$
\begin{aligned}
& G_{n \kappa}(\theta)=n^{-1} \Phi_{n \kappa}^{-1} \sum_{i=1}^{n}\left\{\alpha-1\left[U_{i} \leq P_{\kappa}\left(\mathbf{X}_{i}\right)^{\prime}\left(\theta-\theta_{\kappa 0}\right)-b_{\kappa 0}\left(\mathbf{X}_{i}\right)\right]\right\} P_{\kappa}\left(\mathbf{X}_{i}\right), \\
& G_{n \kappa}^{*}(\theta)=n^{-1} \Phi_{n \kappa}^{-1} \sum_{i=1}^{n}\left\{\alpha-F\left[P_{\kappa}\left(\mathbf{X}_{i}\right)^{\prime}\left(\theta-\theta_{\kappa 0}\right)-b_{\kappa 0}\left(\mathbf{X}_{i}\right) \mid \mathbf{X}_{i}\right]\right\} P_{\kappa}\left(\mathbf{X}_{i}\right),
\end{aligned}
$$

and $\tilde{G}_{n \kappa}(\theta)=G_{n \kappa}(\theta)-G_{n \kappa}^{*}(\theta)$.

Lemma A.3. As $n \rightarrow \infty$,

$$
1_{n}\left\|\tilde{G}_{n \kappa}\left(\theta_{\kappa 0}\right)\right\|=O_{p}\left[(\kappa / n)^{1 / 2}\right] .
$$

Proof. Notice that since the data are i.i.d., $f(\cdot \mid \mathbf{x})$ is bounded away from zero in a neighborhood of zero for all $\mathbf{x}$ (in particular, $f(0 \mid \mathbf{x}) \geq c_{f}$ for all $\mathbf{x}$ ), and the smallest eigenvalue of $\Phi_{n \kappa}$ is bounded away from zero (when $1_{n}=1$ ),

$$
\begin{aligned}
& E\left[1_{n}\left\|\tilde{G}_{n \kappa}\left(\theta_{\kappa 0}\right)\right\|^{2} \mid \mathbf{X}_{1}, \ldots, \mathbf{X}_{n}\right] \\
& \leq 1_{n} n^{-2} \sum_{i=1}^{n}\left[E\left[\left\{F\left[-b_{\kappa 0}\left(\mathbf{X}_{i}\right) \mid \mathbf{X}_{i}\right]-1\left[U \leq-b_{\kappa 0}\left(\mathbf{X}_{i}\right)\right]\right\}^{2} \mid \mathbf{X}_{i}\right] P_{\kappa}\left(\mathbf{X}_{i}\right)^{\prime} \Phi_{n \kappa}^{-2} P_{\kappa}\left(\mathbf{X}_{i}\right)\right] \\
& \leq C 1_{n} n^{-2} \sum_{i=1}^{n} \operatorname{trace}\left[P_{\kappa}\left(\mathbf{X}_{i}\right)^{\prime} \Phi_{n \kappa}^{-2} P_{\kappa}\left(\mathbf{X}_{i}\right)\right] \\
& \leq C 1_{n} n^{-2} \sum_{i=1}^{n} c_{f}^{-1} f\left(0 \mid \mathbf{X}_{i}\right) \operatorname{trace}\left[P_{\kappa}\left(\mathbf{X}_{i}\right)^{\prime} \Phi_{n \kappa}^{-2} P_{\kappa}\left(\mathbf{X}_{i}\right)\right] \\
& \leq C 1_{n} n^{-1} \operatorname{trace}\left\{\Phi_{n \kappa}^{-2}\left\{n^{-1} \sum_{i=1}^{n} f\left(0 \mid \mathbf{X}_{i}\right) P_{\kappa}\left(\mathbf{X}_{i}\right) P_{\kappa}\left(\mathbf{X}_{i}\right)^{\prime}\right]\right\} \\
& =C 1_{n} n^{-1} \operatorname{trace}\left(\Phi_{n \kappa}^{-1}\right) \\
& \leq C n^{-1} d(\kappa) .
\end{aligned}
$$

Therefore, the lemma follows from Markov's inequality.

Lemma A.4. As $n \rightarrow \infty$,

$$
1_{n}\left\|G_{n \kappa}\left(\hat{\theta}_{n \kappa}\right)\right\| \leq C \zeta_{\kappa} \kappa / n \text { almost surely. }
$$


Proof. By Lemma A.2, there is a unique index set $h_{\kappa} \in \mathcal{H}_{\kappa}$ such that $\hat{\theta}_{n \kappa}=\mathbf{P}_{\kappa}\left(h_{\kappa}\right)^{-1} \mathbf{Y}_{\kappa}\left(h_{\kappa}\right)$ almost surely for all sufficiently large $n$. Now write $G_{n \kappa}\left(\hat{\theta}_{n \kappa}\right)=G_{n \kappa 1}\left(\hat{\theta}_{n \kappa}\right)+G_{n \kappa 2}\left(\hat{\theta}_{n \kappa}\right)$, where

$$
G_{n \kappa 1}\left(\hat{\theta}_{n \kappa}\right)=n^{-1} \Phi_{n \kappa}^{-1} \sum_{i=1, i \in h_{\kappa}}^{n}\left\{\alpha-1\left[U_{i} \leq P_{\kappa}\left(\mathbf{X}_{i}\right)^{\prime}\left(\hat{\theta}_{n \kappa}-\theta_{\kappa 0}\right)-b_{\kappa 0}\left(\mathbf{X}_{i}\right)\right]\right\} P_{\kappa}\left(\mathbf{X}_{i}\right),
$$

and

$$
G_{n \kappa 2}\left(\hat{\theta}_{n \kappa}\right)=n^{-1} \Phi_{n \kappa}^{-1} \sum_{i=1, i \in h_{\kappa}^{c}}^{n}\left\{\alpha-1\left[U_{i} \leq P_{\kappa}\left(\mathbf{X}_{i}\right)^{\prime}\left(\hat{\theta}_{n \kappa}-\theta_{\kappa 0}\right)-b_{\kappa 0}\left(\mathbf{X}_{i}\right)\right]\right\} P_{\kappa}\left(\mathbf{X}_{i}\right) .
$$

Notice that $\max _{1 \leq i \leq n} 1_{n}\left\|\Phi_{n \kappa}^{-1} P_{\kappa}\left(\mathbf{X}_{i}\right)\right\| \leq C \max _{1 \leq i \leq n}\left\|P_{\kappa}\left(\mathbf{X}_{i}\right)\right\|=C \zeta_{\kappa}$ for some constant $C<\infty$ since the smallest eigenvalue of $\Phi_{n \kappa}$ is bounded away from zero (when $1_{n}=1$ ). Thus, we have $1_{n}\left\|G_{n \kappa 1}\left(\hat{\theta}_{n \kappa}\right)\right\| \leq C \zeta_{\kappa} d(\kappa) / n$.

Now consider $G_{n \kappa 2}\left(\hat{\theta}_{n \kappa}\right)$. Notice that $G_{n \kappa 2}\left(\hat{\theta}_{n \kappa}\right)^{\prime}=n^{-1} \bar{H}_{n 1 \kappa}\left(\hat{\theta}_{n \kappa}\right) \mathbf{P}_{\kappa}\left(h_{\kappa}\right) \Phi_{n \kappa}^{-1}$. As was explained in the proof of Lemma A.2, each component in $\bar{H}_{n 1 \kappa}\left(\hat{\theta}_{n \kappa}\right)$ is between $\alpha-1$ and $\alpha$. Thus, $\left\|\bar{H}_{n 1 \kappa}\left(\hat{\theta}_{n \kappa}\right)\right\| \leq d(\kappa)^{1 / 2}$. Since the smallest eigenvalue of $\Phi_{n \kappa}$ is bounded away from zero (when $1_{n}=1$ ), we can find a constant $C<\infty$ (independent of $\kappa$ ) such that

$$
1_{n}\left\|\mathbf{P}_{\kappa}\left(h_{\kappa}\right) \Phi_{n \kappa}^{-1}\right\| \leq C\left\|\mathbf{P}_{\kappa}\left(h_{\kappa}\right)\right\|
$$

Also notice that

$$
\begin{aligned}
\left\|\mathbf{P}_{\kappa}\left(h_{\kappa}\right)\right\|^{2} & =\operatorname{trace}\left[\mathbf{P}_{\kappa}\left(h_{\kappa}\right)^{\prime} \mathbf{P}_{\kappa}\left(h_{\kappa}\right)\right]=\operatorname{trace}\left[\mathbf{P}_{\kappa}\left(h_{\kappa}\right) \mathbf{P}_{\kappa}\left(h_{\kappa}\right)^{\prime}\right] \\
& =\sum_{i \in h_{\kappa}}\left\|P_{\kappa}\left(\mathbf{X}_{i}\right)\right\|^{2} \leq \zeta_{\kappa}^{2} d(\kappa) .
\end{aligned}
$$

Hence, $\left\|\mathbf{P}_{\kappa}\left(h_{\kappa}\right)\right\| \leq \zeta_{\kappa} d(\kappa)^{1 / 2}$. Therefore,

$$
1_{n}\left\|G_{n \kappa 2}\left(\hat{\theta}_{n \kappa}\right)\right\| \leq n^{-1}\left\|\bar{H}_{n 1 \kappa}\left(\hat{\theta}_{n \kappa}\right)\right\| 1_{n}\left\|\mathbf{P}_{\kappa}\left(h_{\kappa}\right) \Phi_{n \kappa}^{-1}\right\| \leq C \zeta_{\kappa} d(\kappa) / n
$$

Since arguments used in this proof hold uniformly over $h_{\kappa}$, the lemma follows immediately.

The next lemma is based on the elegant argument of Welsh (1989).

Lemma A.5. As $n \rightarrow \infty$,

$$
\sup _{\left\|\theta-\theta_{\kappa 0}\right\| \leq C(\kappa / n)^{1 / 2}} 1_{n}\left\|\tilde{G}_{n \kappa}(\theta)-\tilde{G}_{n \kappa}\left(\theta_{\kappa 0}\right)\right\|=O_{p}\left[d(\kappa)^{1 / 2} \zeta_{\kappa}^{1 / 2}(d(\kappa) / n)^{3 / 4}(\log n)^{1 / 2}\right] .
$$

Proof. Let $\eta_{n}=(d(\kappa) / n)^{1 / 2}, \gamma_{n}=d(\kappa) / n^{5 / 2}$, and $B_{n}=\left\{\theta:\left\|\theta-\theta_{\kappa 0}\right\| \leq C \eta_{n}\right\}$. As in the proof of Theorem 3.1 of Welsh (1989), cover the ball $B_{n}$ with cubes $\mathcal{C}=\left\{\mathcal{C}\left(\theta_{l}\right)\right\}$, where $\mathcal{C}\left(\theta_{l}\right)$ is a cube 
containing $\theta_{l}$ with sides of length $C\left(d(\kappa) / n^{5}\right)^{1 / 2}$ such that $\theta_{l} \in B_{n}$. Then the number of the cubes covering the ball $B_{n}$ is $L=\left(2 n^{2}\right)^{d(\kappa)}$. Also, we have that $\left\|\theta-\theta_{l}\right\| \leq C \gamma_{n}$ for any $\theta \in \mathcal{C}\left(\theta_{l}\right)$, where $l=1, \cdots, L$.

First note that

$$
\begin{aligned}
& \sup _{\theta \in B_{n}} 1_{n}\left\|\tilde{G}_{n \kappa}(\theta)-\tilde{G}_{n \kappa}\left(\theta_{\kappa 0}\right)\right\| \\
& \leq \max _{1 \leq l \leq L} \sup _{\theta \in \mathcal{C}\left(\theta_{l}\right)} 1_{n}\left\|\tilde{G}_{n \kappa}(\theta)-\tilde{G}_{n \kappa}\left(\theta_{l}\right)\right\|+\max _{1 \leq l \leq L} 1_{n}\left\|\tilde{G}_{n \kappa}\left(\theta_{l}\right)-\tilde{G}_{n \kappa}\left(\theta_{\kappa 0}\right)\right\| .
\end{aligned}
$$

Now using the fact that $1[u \leq \cdot]$ and $F[\cdot \mid \mathbf{x}]$ are monotone increasing functions and that $|A-B| \leq$ $\left|A_{1}-B\right|+\left|A_{2}-B\right|$ for any $A, A_{1}, A_{2}$, and $B$ satisfying $A_{1} \leq A \leq A_{2}$, we have

$$
\begin{aligned}
& \sup _{\theta \in \mathcal{C}\left(\theta_{l}\right)} 1_{n}\left\|\tilde{G}_{n \kappa}(\theta)-\tilde{G}_{n \kappa}\left(\theta_{l}\right)\right\| \\
& \leq \sup _{\theta \in \mathcal{C}\left(\theta_{l}\right)} n^{-1} \sum_{i=1}^{n} 1_{n}\left\|\Phi_{n \kappa}^{-1} P_{\kappa}\left(\mathbf{X}_{i}\right)\right\| \\
& \times \mid\left\{1\left[U_{i} \leq P_{\kappa}\left(\mathbf{X}_{i}\right)^{\prime}\left(\theta-\theta_{\kappa 0}\right)-b_{\kappa 0}\left(\mathbf{X}_{i}\right)\right]-F\left[P_{\kappa}\left(\mathbf{X}_{i}\right)^{\prime}\left(\theta-\theta_{\kappa 0}\right)-b_{\kappa 0}\left(\mathbf{X}_{i}\right) \mid \mathbf{X}_{i}\right]\right\} \\
& -\left\{1\left[U_{i} \leq P_{\kappa}\left(\mathbf{X}_{i}\right)^{\prime}\left(\theta_{l}-\theta_{\kappa 0}\right)-b_{\kappa 0}\left(\mathbf{X}_{i}\right)\right]-F\left[P_{\kappa}\left(\mathbf{X}_{i}\right)^{\prime}\left(\theta_{l}-\theta_{\kappa 0}\right)-b_{\kappa 0}\left(\mathbf{X}_{i}\right) \mid \mathbf{X}_{i}\right]\right\} \mid \\
& \leq n^{-1} \sum_{i=1}^{n} 1_{n}\left\|\Phi_{n \kappa}^{-1} P_{\kappa}\left(\mathbf{X}_{i}\right)\right\| \mid\left\{1\left[U_{i} \leq P_{\kappa}\left(\mathbf{X}_{i}\right)^{\prime}\left(\theta_{l}-\theta_{\kappa 0}\right)-b_{\kappa 0}\left(\mathbf{X}_{i}\right)+\left\|P_{\kappa}\left(\mathbf{X}_{i}\right)\right\| \gamma_{n}\right]\right. \\
& \left.-F\left[P_{\kappa}\left(\mathbf{X}_{i}\right)^{\prime}\left(\theta_{l}-\theta_{\kappa 0}\right)-b_{\kappa 0}\left(\mathbf{X}_{i}\right)-\left\|P_{\kappa}\left(\mathbf{X}_{i}\right)\right\| \gamma_{n} \mid \mathbf{X}_{i}\right]\right\} \\
& -\left\{1\left[U_{i} \leq P_{\kappa}\left(\mathbf{X}_{i}\right)^{\prime}\left(\theta_{l}-\theta_{\kappa 0}\right)-b_{\kappa 0}\left(\mathbf{X}_{i}\right)\right]-F\left[P_{\kappa}\left(\mathbf{X}_{i}\right)^{\prime}\left(\theta_{l}-\theta_{\kappa 0}\right)-b_{\kappa 0}\left(\mathbf{X}_{i}\right) \mid \mathbf{X}_{i}\right]\right\} \mid \\
& +n^{-1} \sum_{i=1}^{n} 1_{n}\left\|\Phi_{n \kappa}^{-1} P_{\kappa}\left(\mathbf{X}_{i}\right)\right\| \mid\left\{1\left[U_{i} \leq P_{\kappa}\left(\mathbf{X}_{i}\right)^{\prime}\left(\theta_{l}-\theta_{\kappa 0}\right)-b_{\kappa 0}\left(\mathbf{X}_{i}\right)-\left\|P_{\kappa}\left(\mathbf{X}_{i}\right)\right\| \gamma_{n}\right]\right. \\
& \left.-F\left[P_{\kappa}\left(\mathbf{X}_{i}\right)^{\prime}\left(\theta_{l}-\theta_{\kappa 0}\right)-b_{\kappa 0}\left(\mathbf{X}_{i}\right)+\left\|P_{\kappa}\left(\mathbf{X}_{i}\right)\right\| \gamma_{n} \mid \mathbf{X}_{i}\right]\right\} \\
& -\left\{1\left[U_{i} \leq P_{\kappa}\left(\mathbf{X}_{i}\right)^{\prime}\left(\theta_{l}-\theta_{\kappa 0}\right)-b_{\kappa 0}\left(\mathbf{X}_{i}\right)\right]-F\left[P_{\kappa}\left(\mathbf{X}_{i}\right)^{\prime}\left(\theta_{l}-\theta_{\kappa 0}\right)-b_{\kappa 0}\left(\mathbf{X}_{i}\right) \mid \mathbf{X}_{i}\right]\right\} \mid \\
& \leq \mid n^{-1} \sum_{i=1}^{n} 1_{n}\left\|\Phi_{n \kappa}^{-1} P_{\kappa}\left(\mathbf{X}_{i}\right)\right\| \\
& \times\left[\left\{1\left[U_{i} \leq P_{\kappa}\left(\mathbf{X}_{i}\right)^{\prime}\left(\theta_{l}-\theta_{\kappa 0}\right)-b_{\kappa 0}\left(\mathbf{X}_{i}\right)+\left\|P_{\kappa}\left(\mathbf{X}_{i}\right)\right\| \gamma_{n}\right]\right.\right. \\
& \left.-F\left[P_{\kappa}\left(\mathbf{X}_{i}\right)^{\prime}\left(\theta_{l}-\theta_{\kappa 0}\right)-b_{\kappa 0}\left(\mathbf{X}_{i}\right)+\left\|P_{\kappa}\left(\mathbf{X}_{i}\right)\right\| \gamma_{n} \mid \mathbf{X}_{i}\right]\right\} \\
& -\left\{1\left[U_{i} \leq P_{\kappa}\left(\mathbf{X}_{i}\right)^{\prime}\left(\theta_{l}-\theta_{\kappa 0}\right)-b_{\kappa 0}\left(\mathbf{X}_{i}\right)-\left\|P_{\kappa}\left(\mathbf{X}_{i}\right)\right\| \gamma_{n}\right]\right. \\
& \left.\left.-F\left[P_{\kappa}\left(\mathbf{X}_{i}\right)^{\prime}\left(\theta_{l}-\theta_{\kappa 0}\right)-b_{\kappa 0}\left(\mathbf{X}_{i}\right)-\left\|P_{\kappa}\left(\mathbf{X}_{i}\right)\right\| \gamma_{n} \mid \mathbf{X}_{i}\right]\right\}\right] \mid
\end{aligned}
$$




$$
\begin{aligned}
& +2 n^{-1} \sum_{i=1}^{n} 1_{n}\left\|\Phi_{n \kappa}^{-1} P_{\kappa}\left(\mathbf{X}_{i}\right)\right\|\left\{F\left[P_{\kappa}\left(\mathbf{X}_{i}\right)^{\prime}\left(\theta_{l}-\theta_{\kappa 0}\right)-b_{\kappa 0}\left(\mathbf{X}_{i}\right)+\left\|P_{\kappa}\left(\mathbf{X}_{i}\right)\right\| \gamma_{n} \mid \mathbf{X}_{i}\right]\right. \\
& \left.-F\left[P_{\kappa}\left(\mathbf{X}_{i}\right)^{\prime}\left(\theta_{l}-\theta_{\kappa 0}\right)-b_{\kappa 0}\left(\mathbf{X}_{i}\right)-\left\|P_{\kappa}\left(\mathbf{X}_{i}\right)\right\| \gamma_{n} \mid \mathbf{X}_{i}\right]\right\} .
\end{aligned}
$$

Consider the second term in (A.2). By Assumption 4.3,

$$
\begin{aligned}
\max _{1 \leq l \leq L} n^{-1} & \sum_{i=1}^{n} 1_{n}\left\|\Phi_{n \kappa}^{-1} P_{\kappa}\left(\mathbf{X}_{i}\right)\right\|\left\{F\left[P_{\kappa}\left(\mathbf{X}_{i}\right)^{\prime}\left(\theta_{l}-\theta_{\kappa 0}\right)-b_{\kappa 0}\left(\mathbf{X}_{i}\right)+\left\|P_{\kappa}\left(\mathbf{X}_{i}\right)\right\| \gamma_{n} \mid \mathbf{X}_{i}\right]\right. \\
& \left.-F\left[P_{\kappa}\left(\mathbf{X}_{i}\right)^{\prime}\left(\theta_{l}-\theta_{\kappa 0}\right)-b_{\kappa 0}\left(\mathbf{X}_{i}\right)-\left\|P_{\kappa}\left(\mathbf{X}_{i}\right)\right\| \gamma_{n} \mid \mathbf{X}_{i}\right]\right\} \\
& \leq C \gamma_{n} \max _{1 \leq i \leq n} 1_{n}\left\|\Phi_{n \kappa}^{-1} P_{\kappa}\left(\mathbf{X}_{i}\right)\right\|\left\|P_{\kappa}\left(\mathbf{X}_{i}\right)\right\| \\
& \leq C \gamma_{n} \zeta_{\kappa}^{2} .
\end{aligned}
$$

Now consider the second term in (A.1), that is $\max _{1 \leq l \leq L} 1_{n}\left\|\tilde{G}_{n \kappa}\left(\theta_{l}\right)-\tilde{G}_{n \kappa}\left(\theta_{\kappa 0}\right)\right\|$. Let $\Delta_{\tilde{G}_{n \kappa}}^{(j)}\left(\theta_{l}\right)$ denote the $j$-th element of $\left[\tilde{G}_{n \kappa}\left(\theta_{l}\right)-\tilde{G}_{n \kappa}\left(\theta_{\kappa 0}\right)\right]$. Then we have

$$
1_{n} \Delta_{\tilde{G}_{n \kappa}}^{(j)}\left(\theta_{l}\right)=1_{n} e_{(j)}^{\prime}\left[\tilde{G}_{n \kappa}\left(\theta_{l}\right)-\tilde{G}_{n \kappa}\left(\theta_{\kappa 0}\right)\right]
$$

where $e_{(j)}$ is a unit vector whose components are all zero except for the $j$-th component being one. Notice that conditional on $\left\{\mathbf{X}_{1}, \ldots, \mathbf{X}_{n}\right\}$, the summands in $1_{n} \Delta_{\tilde{G}_{n \kappa}}^{(j)}\left(\theta_{l}\right)$ are independently distributed with mean 0 and that the summands in $1_{n} \Delta_{\tilde{G}_{n \kappa}}^{(j)}\left(\theta_{l}\right)$ are bounded uniformly (over $j$ and l) by $n^{-1} C \zeta_{\kappa}$ for all sufficiently large $n$. Furthermore, the variance of $1_{n} \Delta_{\tilde{G}_{n \kappa}}^{(j)}\left(\theta_{l}\right)$ conditional on $\left\{\mathbf{X}_{1}, \ldots, \mathbf{X}_{n}\right\}$ is bounded by

$$
C n^{-2} \sum_{i=1}^{n} 1_{n}\left|e_{(j)}^{\prime} \Phi_{n \kappa}^{-1} P_{\kappa}\left(\mathbf{X}_{i}\right)\right|^{2}\left|P_{\kappa}\left(\mathbf{X}_{i}\right)^{\prime}\left(\theta_{l}-\theta_{\kappa 0}\right)\right| .
$$

Notice that using the fact that $f(0 \mid \mathbf{x})$ is bounded away from zero (that is, $f(0 \mid \mathbf{x}) \geq c_{f}$ for all $\mathbf{x}$ ) and that the smallest eigenvalue of $\Phi_{n \kappa}^{-1}$ is bounded away from zero (when $1_{n}=1$ ) for all $\kappa$,

$$
\begin{aligned}
& n^{-1} \sum_{i=1}^{n}\left|e_{(j)}^{\prime} \Phi_{n \kappa}^{-1} P_{\kappa}\left(\mathbf{X}_{i}\right)\right|^{2}\left|P_{\kappa}\left(\mathbf{X}_{i}\right)^{\prime}\left(\theta_{l}-\theta_{\kappa 0}\right)\right| \\
& \leq n^{-1} \sum_{i=1}^{n} c_{f}^{-1} f\left(0 \mid \mathbf{X}_{i}\right)\left|e_{(j)}^{\prime} \Phi_{n \kappa}^{-1} P_{\kappa}\left(\mathbf{X}_{i}\right)\right|^{2}\left|P_{\kappa}\left(\mathbf{X}_{i}\right)^{\prime}\left(\theta_{l}-\theta_{\kappa 0}\right)\right| \\
& \leq C \max _{1 \leq i \leq n}\left|P_{\kappa}\left(\mathbf{X}_{i}\right)^{\prime}\left(\theta_{l}-\theta_{\kappa 0}\right)\right| e_{(j)}^{\prime} \Phi_{n \kappa}^{-1}\left[n^{-1} \sum_{i=1}^{n} f\left(0 \mid \mathbf{X}_{i}\right) P_{\kappa}\left(\mathbf{X}_{i}\right) P_{\kappa}\left(\mathbf{X}_{i}\right)^{\prime}\right] \Phi_{n \kappa}^{-1} e_{(j)} \\
& \leq C \zeta_{\kappa} \eta_{n} \lambda_{\max }\left(\Phi_{n \kappa}^{-1}\right) \\
& \leq C \zeta_{\kappa} \eta_{n}
\end{aligned}
$$

uniformly (over $j$ and $l$ ) for all sufficiently large $n$. Therefore, the conditional variance of $1_{n} \Delta_{\tilde{G}_{n \kappa}}^{(j)}\left(\theta_{l}\right)$ is bounded uniformly (over $j$ and $l$ ) by $n^{-1} C \zeta_{\kappa} \eta_{n}$ for all sufficiently large $n$. 
Let $\varepsilon_{n}=d(\kappa)^{1 / 2} \zeta_{\kappa}^{1 / 2}(d(\kappa) / n)^{3 / 4}(\log n)^{1 / 2}$. An application of Bernstein's inequality (see, for example, van der Vaart and Wellner $\left(1996\right.$, p.102)) to the sum $\Delta_{\tilde{G}_{n \kappa}}^{(j)}\left(\theta_{l}\right)$ gives

$$
\begin{aligned}
& \operatorname{Pr}\left(\max _{1 \leq l \leq L} 1_{n}\left\|\tilde{G}_{n \kappa}\left(\theta_{l}\right)-\tilde{G}_{n \kappa}\left(\theta_{\kappa 0}\right)\right\|>C \varepsilon_{n} \mid \mathbf{X}_{1}, \ldots, \mathbf{X}_{n}\right) \\
& \leq \sum_{l=1}^{L} \operatorname{Pr}\left(1_{n}\left\|\tilde{G}_{n \kappa}\left(\theta_{l}\right)-\tilde{G}_{n \kappa}\left(\theta_{\kappa 0}\right)\right\|>C \varepsilon_{n} \mid \mathbf{X}_{1}, \ldots, \mathbf{X}_{n}\right) \\
& \leq \sum_{l=1}^{L} \sum_{j=1}^{d(\kappa)} \operatorname{Pr}\left(1_{n}\left|\Delta_{\tilde{G}_{n \kappa}^{(j)}}\left(\theta_{l}\right)\right|>C \varepsilon_{n} d(\kappa)^{-1 / 2} \mid \mathbf{X}_{1}, \ldots, \mathbf{X}_{n}\right) \\
& \leq 2\left(2 n^{2}\right)^{d(\kappa)} d(\kappa) \exp \left[-C \frac{\varepsilon_{n}^{2} d(\kappa)^{-1}}{n^{-1} \zeta_{\kappa} \eta_{n}+n^{-1} \zeta_{\kappa} \varepsilon_{n} d(\kappa)^{-1 / 2}}\right] \\
& \leq C \exp [2 d(\kappa) \log n+\log d(\kappa)-C d(\kappa) \log n] \\
& \leq C \exp [-C d(\kappa) \log n]
\end{aligned}
$$

for all sufficiently large $n$. In particular, it is required here that $\zeta_{\kappa}=O\left(\kappa^{1 / 2}\right)$ and $\left(\kappa^{2} / n\right)(\log n)^{2} \rightarrow 0$.

Now consider the first term in (A.2). Let $\tilde{T}_{n \kappa}\left(\theta_{l}\right)$ denote the expression inside $|\cdot|$ in the first term in (A.2). Notice that conditional on $\left\{\mathbf{X}_{1}, \ldots, \mathbf{X}_{n}\right\}$, the summands in $\tilde{T}_{n}\left(\theta_{l}\right)$ are independently distributed with mean 0 and with range bounded by $n^{-1} C \zeta_{\kappa}$ and that the variance of the summands in $\tilde{T}_{n \kappa}\left(\theta_{l}\right)$ conditional on $\left\{\mathbf{X}_{1}, \ldots, \mathbf{X}_{n}\right\}$ is bounded by $n^{-1} C \zeta_{\kappa}^{3} \gamma_{n}$ uniformly over $l$ for all sufficiently large $n$. Another application of Bernstein's inequality to $\tilde{T}_{n}\left(\theta_{l}\right)$ gives

$$
\begin{aligned}
\operatorname{Pr}\left(\max _{1 \leq l \leq L}\left|\tilde{T}_{n \kappa}\left(\theta_{l}\right)\right|>C \varepsilon_{n} \mid \mathbf{X}_{1}, \ldots, \mathbf{X}_{n}\right) & \leq \sum_{l=1}^{L} \operatorname{Pr}\left(\left|\tilde{T}_{n \kappa}\left(\theta_{l}\right)\right|>C \varepsilon_{n} \mid \mathbf{X}_{1}, \ldots, \mathbf{X}_{n}\right) \\
& \leq 2\left(2 n^{2}\right)^{d(\kappa)} \exp \left[-C \frac{\varepsilon_{n}^{2}}{n^{-1} \gamma_{n} \zeta_{\kappa}^{3}+n^{-1} \zeta_{\kappa} \varepsilon_{n}}\right] \\
& \leq 2\left(2 n^{2}\right)^{d(\kappa)} \exp \left[-C n \varepsilon_{n} / \zeta_{\kappa}\right] \\
& \leq C \exp \left[2 d(\kappa) \log n+\log d(\kappa)-C d(\kappa) n^{1 / 4}(\log n)^{1 / 2}\right]
\end{aligned}
$$

for all sufficiently large $n$. Now the lemma follows by combining (A.3), (A.4), and (A.5).

Lemma A.6. As $n \rightarrow \infty$,

$$
\sup _{\left\|\theta-\theta_{\kappa 0}\right\| \leq C(\kappa / n)^{1 / 2}} 1_{n}\left\|G_{n \kappa}(\theta)-G_{n \kappa}^{*}(\theta)\right\|=O_{p}\left[(d(\kappa) / n)^{1 / 2}\right] .
$$

Proof. Using triangle inequality, write

$$
\begin{aligned}
& \sup _{\left\|\theta-\theta_{\kappa 0}\right\| \leq C(\kappa / n)^{1 / 2}} 1_{n}\left\|G_{n \kappa}(\theta)-G_{n \kappa}^{*}(\theta)\right\| \\
& \leq \sup _{\left\|\theta-\theta_{\kappa 0}\right\| \leq C(\kappa / n)^{1 / 2}} 1_{n}\left\|\tilde{G}_{n \kappa}(\theta)-\tilde{G}_{n \kappa}\left(\theta_{\kappa 0}\right)\right\|+1_{n}\left\|\tilde{G}_{n \kappa}\left(\theta_{\kappa 0}\right)\right\| .
\end{aligned}
$$

Then the desired result follows immediately from Lemmas A.3 and A.5. 
Lemma A.7. As $n \rightarrow \infty$,

$$
1_{n} G_{n \kappa}^{*}(\theta)=-1_{n}\left(\theta-\theta_{\kappa 0}\right)+1_{n} n^{-1} \Phi_{n \kappa}^{-1} \sum_{i=1}^{n} f\left(0 \mid \mathbf{X}_{i}\right) P_{\kappa}\left(\mathbf{X}_{i}\right) b_{\kappa 0}\left(\mathbf{X}_{i}\right)+R_{n \kappa}^{*},
$$

where $\left\|R_{n \kappa}^{*}\right\|=O\left[\zeta_{\kappa}\left\|\theta-\theta_{\kappa 0}\right\|^{2}+\zeta_{\kappa} \kappa^{-2 r}\right]$.

Proof. Define

$$
1_{n} \tilde{G}_{n \kappa}^{*}(\theta)=-1_{n}\left(\theta-\theta_{\kappa 0}\right)+1_{n} n^{-1} \Phi_{n \kappa}^{-1} \sum_{i=1}^{n} f\left(0 \mid \mathbf{X}_{i}\right) P_{\kappa}\left(\mathbf{X}_{i}\right) b_{\kappa 0}\left(\mathbf{X}_{i}\right) .
$$

Using a first-order Taylor series expansion, Assumptions 4.3 and 4.5, and equation 4.2, we have

$$
\begin{aligned}
1_{n}\left\|G_{n \kappa}^{*}(\theta)-\tilde{G}_{n \kappa}^{*}(\theta)\right\| & \leq C \max _{1 \leq i \leq n} 1_{n}\left\|\Phi_{n \kappa}^{-1} P_{\kappa}\left(\mathbf{X}_{i}\right)\right\|\left[n^{-1} \sum_{i=1}^{n}\left\{P_{\kappa}\left(\mathbf{X}_{i}\right)^{\prime}\left(\theta-\theta_{\kappa 0}\right)-b_{\kappa 0}\left(\mathbf{X}_{i}\right)\right\}^{2}\right] \\
& \leq C \zeta_{\kappa}\left\{\left(\theta-\theta_{\kappa 0}\right)^{\prime} \Phi_{n \kappa}\left(\theta-\theta_{\kappa 0}\right)+\max _{1 \leq i \leq n} b_{\kappa 0}\left(\mathbf{X}_{i}\right)^{2}\right\} \\
& \leq C \zeta_{\kappa} \lambda_{\max }\left(\Phi_{n \kappa}\right)\left(\theta-\theta_{\kappa 0}\right)^{\prime}\left(\theta-\theta_{\kappa 0}\right)+C \zeta_{\kappa} \max _{1 \leq i \leq n} b_{\kappa 0}\left(\mathbf{X}_{i}\right)^{2} \\
& \leq O\left[\zeta_{\kappa}\left\|\theta-\theta_{\kappa 0}\right\|^{2}\right]+O\left(\zeta_{\kappa} \kappa^{-2 r}\right)
\end{aligned}
$$

for all sufficiently large $n$, which proves the lemma.

Lemma A.8. As $\kappa \rightarrow \infty$,

$$
1_{n}\left\|n^{-1} \Phi_{n \kappa}^{-1} \sum_{i=1}^{n} f\left(0 \mid \mathbf{X}_{i}\right) P_{\kappa}\left(\mathbf{X}_{i}\right) b_{\kappa 0}\left(\mathbf{X}_{i}\right)\right\|=O\left(\kappa^{-r}\right) .
$$

Proof. Let $\bar{B}_{\kappa}$ be a $(n \times 1)$ vector whose elements are $f\left(0 \mid \mathbf{X}_{i}\right)^{1 / 2} b_{\kappa 0}\left(\mathbf{X}_{i}\right)$ and $\overline{\mathbf{P}}_{\kappa}$ be a $(n \times d(\kappa))$ matrix whose rows are $f\left(0 \mid \mathbf{X}_{i}\right)^{1 / 2} P_{\kappa}\left(\mathbf{X}_{i}\right)^{\prime}$. Then $\Phi_{n \kappa}=\overline{\mathbf{P}}_{\kappa}^{\prime} \overline{\mathbf{P}}_{\kappa} / n$ and

$$
\sum_{i=1}^{n} f\left(0 \mid \mathbf{X}_{i}\right) P_{\kappa}\left(\mathbf{X}_{i}\right) b_{\kappa 0}\left(\mathbf{X}_{i}\right)=\overline{\mathbf{P}}_{\kappa}^{\prime} \bar{B}_{\kappa}
$$

Therefore, using the fact that $\overline{\mathbf{P}}_{\kappa}\left(\overline{\mathbf{P}}_{\kappa}^{\prime} \overline{\mathbf{P}}_{\kappa}\right)^{-1} \overline{\mathbf{P}}_{\kappa}^{\prime}$ is idempotent (so that its largest eigenvalue is just one),

$$
\begin{aligned}
& 1_{n}\left\|n^{-1} \Phi_{n \kappa}^{-1} \sum_{i=1}^{n} f\left(0 \mid \mathbf{X}_{i}\right) P_{\kappa}\left(\mathbf{X}_{i}\right) b_{\kappa 0}\left(\mathbf{X}_{i}\right)\right\|^{2} \\
& =1_{n}\left\|\Phi_{n \kappa}^{-1} \overline{\mathbf{P}}_{\kappa}^{\prime} \bar{B}_{\kappa} / n\right\|^{2} \\
& =1_{n} n^{-2} \bar{B}_{\kappa}^{\prime} \overline{\mathbf{P}}_{\kappa} \Phi_{n \kappa}^{-2} \overline{\mathbf{P}}_{\kappa}^{\prime} \bar{B}_{\kappa} \\
& \leq 1_{n} n^{-2} \lambda_{\max }\left(\Phi_{n \kappa}^{-1}\right) \bar{B}_{\kappa}^{\prime} \overline{\mathbf{P}}_{\kappa}\left(\overline{\mathbf{P}}_{\kappa}^{\prime} \overline{\mathbf{P}}_{\kappa} / n\right)^{-1} \overline{\mathbf{P}}_{\kappa}^{\prime} \bar{B}_{\kappa} \\
& \leq 1_{n} n^{-1} \lambda_{\max }\left(\Phi_{n \kappa}^{-1}\right) \lambda_{\max }\left[\overline{\mathbf{P}}_{\kappa}\left(\overline{\mathbf{P}}_{\kappa}^{\prime} \overline{\mathbf{P}}_{\kappa}\right)^{-1} \overline{\mathbf{P}}_{\kappa}^{\prime}\right]\left\|\bar{B}_{\kappa}\right\|^{2} \\
& \leq C \max _{1 \leq i \leq n} b_{\kappa 0}\left(\mathbf{X}_{i}\right)^{2}
\end{aligned}
$$

for all sufficiently large $n$. The lemma now follows from equation (4.2). 
Proof of Theorem 4.1. We will obtain the rate of convergence using 'convexity' arguments similar to those used in the proof of Theorem 1 of He and Shi (1998). Define $M_{n \kappa}(\theta)=-\left(\theta-\theta_{\kappa 0}\right)^{\prime} G_{n \kappa}(\theta)$. Notice that $M_{n \kappa}(\theta)$ is a convex function, thereby implying that

$$
\left\|G_{n \kappa}(\theta)\right\| \geq M_{n \kappa}\left[t\left(\theta-\theta_{\kappa 0}\right)+\theta_{\kappa 0}\right] / t\left\|\theta-\theta_{\kappa 0}\right\|
$$

for any $t \geq 1$. Also, notice that the right-hand side of inequality (A.7) is weakly increasing in $t$. Let $\eta_{n}=(\kappa / n)^{1 / 2}$. As in equation (A.4) of He and Shi (1998), for any $\theta$,

$$
\begin{aligned}
& \inf _{\left\|\theta-\theta_{\kappa 0}\right\| \geq C \eta_{n}} 1_{n}\left\|G_{n \kappa}(\theta)\right\| \\
& \geq \inf _{\left\|\theta-\theta_{\kappa 0}\right\|=C \eta_{n}} \inf _{t \geq 1} 1_{n} M_{n \kappa}\left[t\left(\theta-\theta_{\kappa 0}\right)+\theta_{\kappa 0}\right] / t\left\|\theta-\theta_{\kappa 0}\right\| \\
& \geq \inf _{\left\|\theta-\theta_{\kappa 0}\right\|=C \eta_{n}} 1_{n} M_{n \kappa}(\theta) /\left\|\theta-\theta_{\kappa 0}\right\| \\
& =\inf _{\left\|\theta-\theta_{\kappa 0}\right\|=C \eta_{n}}-\left(\theta-\theta_{\kappa 0}\right)^{\prime} 1_{n}\left\{G_{n \kappa}^{*}(\theta)+\left[G_{n \kappa}(\theta)-G_{n \kappa}^{*}(\theta)\right]\right\} /\left\|\theta-\theta_{\kappa 0}\right\| \\
& =\inf _{\left\|\theta-\theta_{\kappa 0}\right\|=C \eta_{n}}\left(\theta-\theta_{\kappa 0}\right)^{\prime}\left(\theta-\theta_{\kappa 0}\right) /\left\|\theta-\theta_{\kappa 0}\right\|+\eta_{n} O_{p}(1)+O\left(\kappa^{-r}\right),
\end{aligned}
$$

where the last equality follows from Lemmas A.6, A.7, and A.8. In view of this and Lemma A.4, for any $\varepsilon>0$ and any positive constant $C$,

$$
\begin{aligned}
& \operatorname{Pr}\left(\left\|\hat{\theta}_{n \kappa}-\theta_{\kappa 0}\right\| \geq C \eta_{n}\right) \\
& \leq \operatorname{Pr}\left(\left\|\hat{\theta}_{n \kappa}-\theta_{\kappa 0}\right\| \geq C \eta_{n}, 1_{n}\left\|G_{n \kappa}\left(\hat{\theta}_{n \kappa}\right)\right\|<C \eta_{n}\right)+\operatorname{Pr}\left(1_{n}\left\|G_{n \kappa}\left(\hat{\theta}_{n \kappa}\right)\right\| \geq C \eta_{n}\right) \\
& <\varepsilon
\end{aligned}
$$

provided that $\kappa^{2} / n \rightarrow 0$. This implies that $\left\|\hat{\theta}_{n \kappa}-\theta_{\kappa 0}\right\|=O_{p}\left[(\kappa / n)^{1 / 2}\right]$, which proves part (a). Part (b) follows by combining part (a) with $\zeta_{\kappa}=O\left(\kappa^{1 / 2}\right)$ since

$$
\sup _{\mathbf{x} \in \mathcal{X}}|\tilde{m}(\mathbf{x})-m(\mathbf{x})| \leq \sup _{\mathbf{x} \in \mathcal{X}}\left\|P_{\kappa}(\mathbf{x})\right\|\left\|\hat{\theta}_{n \kappa}-\theta_{\kappa 0}\right\| .
$$

Proof of Theorem 4.2. Write

$$
1_{n} G_{n \kappa}\left(\hat{\theta}_{n \kappa}\right)=1_{n} \tilde{G}_{n \kappa}\left(\theta_{\kappa 0}\right)+1_{n}\left[\tilde{G}_{n \kappa}\left(\hat{\theta}_{n \kappa}\right)-\tilde{G}_{n \kappa}\left(\theta_{\kappa 0}\right)\right]+1_{n} G_{n \kappa}^{*}\left(\hat{\theta}_{n \kappa}\right) .
$$

By Lemma A.7, (A.8) can be rewritten as

$$
\begin{aligned}
1_{n}\left(\hat{\theta}_{n \kappa}-\theta_{\kappa 0}\right) & =-1_{n} G_{n \kappa}\left(\hat{\theta}_{n \kappa}\right)+1_{n} \tilde{G}_{n \kappa}\left(\theta_{\kappa 0}\right)+1_{n}\left[\tilde{G}_{n \kappa}\left(\hat{\theta}_{n \kappa}\right)-\tilde{G}_{n \kappa}\left(\theta_{\kappa 0}\right)\right] \\
& +1_{n} n^{-1} \Phi_{n \kappa}^{-1} \sum_{i=1}^{n} f\left(0 \mid \mathbf{X}_{i}\right) P_{\kappa}\left(\mathbf{X}_{i}\right) b_{\kappa 0}\left(\mathbf{X}_{i}\right)+R_{n \kappa}^{*} .
\end{aligned}
$$


By applying Lemmas A.4, A.5, and A.7, and Theorem 4.1 (a) to (A.9), we have

$$
1_{n}\left(\hat{\theta}_{n \kappa}-\theta_{\kappa 0}\right)=1_{n} \tilde{G}_{n \kappa}\left(\theta_{\kappa 0}\right)+1_{n} n^{-1} \Phi_{n \kappa}^{-1} \sum_{i=1}^{n} f\left(0 \mid \mathbf{X}_{i}\right) P_{\kappa}\left(\mathbf{X}_{i}\right) b_{\kappa 0}\left(\mathbf{X}_{i}\right)+R_{n}
$$

where the remainder term $R_{n}$ satisfies

$$
\left\|R_{n}\right\|=O_{p}\left[\left(\kappa^{2} / n\right)^{3 / 4}(\log n)^{1 / 2}+\kappa^{3 / 2} / n\right] .
$$

Define

$$
\bar{G}_{n \kappa}\left(\theta_{\kappa 0}\right)=n^{-1} \Phi_{n \kappa}^{-1} \sum_{i=1}^{n}\left\{\alpha-1\left[U_{i} \leq 0\right]\right\} P_{\kappa}\left(\mathbf{X}_{i}\right) .
$$

By using arguments similar to those used in the proof of Lemma A.3, we have

$$
E\left[1_{n}\left\|\tilde{G}_{n \kappa}\left(\theta_{\kappa 0}\right)-\bar{G}_{n \kappa}\left(\theta_{\kappa 0}\right)\right\|^{2} \mid \mathbf{X}_{1}, \ldots, \mathbf{X}_{n}\right] \leq C n^{-1} d(\kappa) \sup _{\mathbf{x} \in \mathcal{X}}\left|b_{\kappa 0}(\mathbf{x})\right|
$$

Hence,

$$
1_{n}\left\|\tilde{G}_{n \kappa}\left(\theta_{\kappa 0}\right)-\bar{G}_{n \kappa}\left(\theta_{\kappa 0}\right)\right\|=o_{p}\left(n^{-1 / 2}\right)
$$

by Markov's inequality. The theorem now follows from the fact that $\left\|\Phi_{n \kappa}-\Phi_{\kappa}\right\|=O_{p}\left(\kappa^{2} / n\right)=o_{p}(1)$ and $\operatorname{Pr}\left(1_{n}=1\right) \rightarrow 1$ as $n \rightarrow \infty$.

\section{A.2 Proof of Theorem 4.3}

We need additional notation to prove Theorem 4.3. Recall that $D^{k} m_{j}\left(x^{j}\right)$ denotes the $k$-th order derivative of $m_{j}$. Define

$$
\begin{aligned}
\beta_{n}\left(x^{1}\right) & =\left[m_{1}\left(x^{1}\right), \delta_{n} D^{1} m_{1}\left(x^{1}\right), \ldots, \delta_{n}^{r-1} D^{(r-1)} m_{1}\left(x^{1}\right)\{(r-1) !\}^{-1}\right]^{\prime}, \\
Z_{n i}\left(x^{1}\right) & =\left[1, \delta_{n}^{-1}\left(X_{i}^{1}-x^{1}\right), \ldots,\left\{\delta_{n}^{-1}\left(X_{i}^{1}-x^{1}\right)\right\}^{(r-1)}\right]^{\prime}, \\
K_{n i}\left(x^{1}\right) & =K\left(\frac{x^{1}-X_{i}^{1}}{\delta_{n}}\right), \text { and } \\
B_{i}\left(x^{1}\right) & =m_{1}\left(X_{i}^{1}\right)-m_{1}\left(x^{1}\right)-\sum_{k=1}^{(r-1)}\{k !\}^{-1} D^{k} m_{1}\left(x^{1}\right)\left(X_{i}^{1}-x^{1}\right) .
\end{aligned}
$$

To simplify the notation, dependence on $x^{1}$ of $\beta_{n}\left(x^{1}\right), Z_{n i}\left(x^{1}\right), K_{n i}\left(x^{1}\right)$, and $B_{i}\left(x^{1}\right)$ will be suppressed throughout the proof (when there is no confusion). For example, $B_{i}=m_{1}\left(X_{i}^{1}\right)-Z_{n i}^{\prime} \beta_{n}$. Also, define

$$
\bar{b}_{\kappa 0}(\tilde{\mathbf{x}})=\mu+m_{-1}(\tilde{\mathbf{x}})-\bar{P}_{\kappa}(\tilde{\mathbf{x}})^{\prime} \theta_{\kappa 0} .
$$


where $\bar{P}_{\kappa}(\tilde{\mathbf{x}})$ is defined in the main text. Recall that

$$
\bar{P}_{\kappa}(\tilde{\mathbf{x}})=\left[1,0, \ldots, 0, p_{1}\left(x^{2}\right), \ldots, p_{\kappa}\left(x^{2}\right), \ldots, p_{1}\left(x^{d}\right), \ldots, p_{\kappa}\left(x^{d}\right)\right]^{\prime} .
$$

Then $\tilde{\mu}+\tilde{m}_{-1}\left(\tilde{\mathbf{X}}_{i}\right)=\bar{P}_{\kappa}\left(\tilde{\mathbf{X}}_{i}\right)^{\prime} \hat{\theta}_{n \kappa}$ and $[\tilde{\mu}-\mu]+\left[\tilde{m}_{-1}\left(\tilde{\mathbf{X}}_{i}\right)-m_{-1}\left(\tilde{\mathbf{X}}_{i}\right)\right]=\bar{P}_{\kappa}\left(\tilde{\mathbf{X}}_{i}\right)^{\prime}\left(\hat{\theta}_{n \kappa}-\theta_{\kappa 0}\right)-\bar{b}_{\kappa 0}\left(\tilde{\mathbf{X}}_{i}\right)$.

Finally, define

$$
\begin{aligned}
G_{n}\left(\mathbf{b}, x^{1}\right) & =\left(n \delta_{n}\right)^{-1} \sum_{i=1}^{n}\left\{\alpha-1\left[U_{i} \leq Z_{n i}^{\prime}\left(\mathbf{b}-\beta_{n}\right)-B_{i}\right]\right\} Z_{n i} K_{n i}, \\
\tilde{G}_{n}\left(\mathbf{b}, x^{1}\right) & =\left(n \delta_{n}\right)^{-1} \sum_{i=1}^{n}\left\{\alpha-1\left[U_{i} \leq Z_{n i}^{\prime}\left(\mathbf{b}-\beta_{n}\right)-B_{i}+\bar{P}_{\kappa}\left(\tilde{\mathbf{X}}_{i}\right)^{\prime}\left(\hat{\theta}_{n \kappa}-\theta_{\kappa 0}\right)-\bar{b}_{\kappa 0}\left(\tilde{\mathbf{X}}_{i}\right)\right]\right\} Z_{n i} K_{n i}, \\
G_{n}^{*}\left(\mathbf{b}, x^{1}\right) & =\left(n \delta_{n}\right)^{-1} \sum_{i=1}^{n}\left\{\alpha-F\left[Z_{n i}^{\prime}\left(\mathbf{b}-\beta_{n}\right)-B_{i} \mid \mathbf{X}_{i}\right]\right\} Z_{n i} K_{n i}, \\
\tilde{G}_{n}^{*}\left(\mathbf{b}, x^{1}\right) & =\left(n \delta_{n}\right)^{-1} \sum_{i=1}^{n}\left\{\alpha-F\left[Z_{n i}^{\prime}\left(\mathbf{b}-\beta_{n}\right)-B_{i}+\bar{P}_{\kappa}\left(\tilde{\mathbf{X}}_{i}\right)^{\prime}\left(\hat{\theta}_{n \kappa}-\theta_{\kappa 0}\right)-\bar{b}_{\kappa 0}\left(\tilde{\mathbf{X}}_{i}\right) \mid \mathbf{X}_{i}\right]\right\} Z_{n i} K_{n i}, \\
\Delta_{G_{n}}\left(\mathbf{b}, x^{1}\right) & =G_{n}\left(\mathbf{b}, x^{1}\right)-G_{n}^{*}\left(\mathbf{b}, x^{1}\right), \text { and } \Delta_{\tilde{G}_{n}}\left(\mathbf{b}, x^{1}\right)=\tilde{G}_{n}\left(\mathbf{b}, x^{1}\right)-\tilde{G}_{n}^{*}\left(\mathbf{b}, x^{1}\right) .
\end{aligned}
$$

The following lemmas are useful to prove Theorem 4.3.

Lemma A.9. As $n \rightarrow \infty$, for any $x^{1}$ such that $\left|x^{1}\right| \leq 1-\delta_{n}$,

$$
\left\|\tilde{G}_{n}\left(\hat{\mathbf{b}}_{n}, x^{1}\right)\right\|=O\left[\left(n \delta_{n}\right)^{-1}\right] \quad \text { almost surely. }
$$

Proof. Notice that the minimization problem (2.2) is just a kernel-weighted linear quantile regression problem and therefore, it has a linear programming representation. Also, notice that each component of $Z_{n i}$ is bounded by one whenever $K_{n i}$ is nonzero. Then the lemma can be proved by using arguments identical to those used in the proof of Lemma A.4.

Lemma A.10. As $n \rightarrow \infty$, for any $x^{1}$ such that $\left|x^{1}\right| \leq 1-\delta_{n}$,

$$
\left\|\Delta_{G_{n}}\left(\beta_{n}, x^{1}\right)\right\|=O_{p}\left[\left(n \delta_{n}\right)^{-1 / 2}\right] .
$$

Proof. Notice that the mean of $\Delta_{G_{n}}\left(\beta_{n}, x^{1}\right)$ is zero. Then the lemma follows by calculating $E\left[\left\|\Delta_{G_{n}}\left(\beta_{n}, x^{1}\right)\right\|^{2}\right]$ and then applying Markov's inequality.

Lemma A.11. As $n \rightarrow \infty$, for any $x^{1}$ such that $\left|x^{1}\right| \leq 1-\delta_{n}$,

$$
\sup _{\left\|\mathbf{b}-\beta_{n}\right\| \leq C\left(n \delta_{n}\right)^{-1 / 2}}\left\|\Delta_{G_{n}}\left(\mathbf{b}, x^{1}\right)-\Delta_{G_{n}}\left(\beta_{n}, x^{1}\right)\right\|=O_{p}\left[\left(n \delta_{n}\right)^{-3 / 4}(\log n)^{1 / 2}\right] .
$$


Proof. The proof of Lemma A.11 is analogous to that of Lemma A.5. Let $\tilde{B}_{n}=\left\{\mathbf{b}:\left\|\mathbf{b}-\beta_{n}\right\| \leq\right.$ $\left.C\left(n \delta_{n}\right)^{-1 / 2}\right\}$. As in the proofs of Lemma A.5 and Theorem 3.1 of Welsh (1989), cover the ball $\tilde{B}_{n}$ with cubes $\tilde{\mathcal{C}}=\left\{\mathcal{C}\left(\mathbf{b}_{l}\right)\right\}$, where $\mathcal{C}\left(\mathbf{b}_{l}\right)$ is a cube containing $\mathbf{b}_{l}$ with sides of $C\left(n^{5} \delta_{n}\right)^{-1 / 2}$ such that $\mathbf{b}_{l} \in \tilde{B}_{n}$. Then the number of the cubes covering the ball $\tilde{B}_{n}$ is $\tilde{L}=\left(2 n^{2}\right)^{r}$. Also, we have that $\left\|\left(\mathbf{b}-\beta_{n}\right)-\left(\mathbf{b}_{l}-\beta_{n}\right)\right\| \leq \sqrt{r} C\left(n^{5} \delta_{n}\right)^{-1 / 2} \equiv \tilde{\gamma}_{n}$ for any $\mathbf{b} \in \mathcal{C}\left(\mathbf{b}_{l}\right)$, where $l=1, \cdots, \tilde{L}$.

As in the proof of Lemma A.5 (in particular, equations (A.1) and (A.2)),

$$
\begin{aligned}
& \sup _{\mathbf{b} \in \tilde{B}_{n}}\left\|\Delta_{G_{n}}\left(\mathbf{b}, x^{1}\right)-\Delta_{G_{n}}\left(\beta_{n}, x^{1}\right)\right\| \\
& \leq \max _{1 \leq l \leq \tilde{L}} \sup _{\left(\mathbf{b}-\beta_{n}\right) \in \mathcal{C}\left(\mathbf{b}_{l}\right)}\left\|\Delta_{G_{n}}\left(\mathbf{b}, x^{1}\right)-\Delta_{G_{n}}\left(\mathbf{b}_{l}, x^{1}\right)\right\|+\max _{1 \leq l \leq \tilde{L}}\left\|\Delta_{G_{n}}\left(\mathbf{b}_{l}, x^{1}\right)-\Delta_{G_{n}}\left(\beta_{n}, x^{1}\right)\right\| \\
& \leq \max _{1 \leq l \leq \tilde{L}}\left\|\Delta_{G_{n}}\left(\mathbf{b}_{l}, x^{1}\right)-\Delta_{G_{n}}\left(\beta_{n}, x^{1}\right)\right\| \\
& +\max _{1 \leq l \leq \tilde{L}} \mid\left(n \delta_{n}\right)^{-1} \sum_{i=1}^{n}\left\|Z_{n i} K_{n i}\right\| \\
& \quad \times\left[\left\{1\left[U_{i} \leq Z_{n i}^{\prime}\left(\mathbf{b}_{l}-\beta_{n}\right)-B_{i}+\left\|Z_{n i}\right\| \tilde{\gamma}_{n}\right]-F\left[Z_{n i}^{\prime}\left(\mathbf{b}_{l}-\beta_{n}\right)-B_{i}+\left\|Z_{n i}\right\| \tilde{\gamma}_{n} \mid \mathbf{X}_{i}\right]\right\}\right. \\
& \left.\quad-\left\{1\left[U_{i} \leq Z_{n i}^{\prime}\left(\mathbf{b}_{l}-\beta_{n}\right)-B_{i}-\left\|Z_{n i}\right\| \tilde{\gamma}_{n}\right]-F\left[Z_{n i}^{\prime}\left(\mathbf{b}_{l}-\beta_{n}\right)-B_{i}-\left\|Z_{n i}\right\| \tilde{\gamma}_{n} \mid \mathbf{X}_{i}\right]\right\}\right] \mid \\
& +2 \max _{1 \leq l \leq \tilde{L}}\left(n \delta_{n}\right)^{-1} \sum_{i=1}^{n}\left\|Z_{n i} K_{n i}\right\|\left\{F\left[Z_{n i}^{\prime}\left(\mathbf{b}_{l}-\beta_{n}\right)-B_{i}+\left\|Z_{n i}\right\| \tilde{\gamma}_{n} \mid \mathbf{X}_{i}\right]\right. \\
& \left.\quad-F\left[Z_{n i}^{\prime}\left(\mathbf{b}_{l}-\beta_{n}\right)-B_{i}-\left\|Z_{n i}\right\| \tilde{\gamma}_{n} \mid \mathbf{X}_{i}\right]\right\} .
\end{aligned}
$$

Now with some modifications, arguments similar to those in the proof of Lemma A.5 yield the desired result.

Lemma A.12. As $n \rightarrow \infty$, for any $x^{1}$ such that $\left|x^{1}\right| \leq 1-\delta_{n}$,

$$
\left\|\Delta_{\tilde{G}_{n}}\left(\mathbf{b}, x^{1}\right)-\Delta_{G_{n}}\left(\mathbf{b}, x^{1}\right)\right\|=O_{p}\left[\left(n \delta_{n}\right)^{-1 / 2}\left[\kappa^{2} / n\right]^{1 / 4}(\log n)^{1 / 2}\right]
$$

for any $\mathbf{b}$ satisfying $\left\|\mathbf{b}-\beta_{n}\right\| \leq C\left(n \delta_{n}\right)^{-1 / 2}$.

Proof. To prove the lemma, define

$$
\begin{aligned}
\tilde{H}_{n}\left(\mathbf{b}, x^{1}, \theta\right) & =\left(n \delta_{n}\right)^{-1} \sum_{i=1}^{n}\left\{\alpha-1\left[U_{i} \leq Z_{n i}^{\prime}\left(\mathbf{b}-\beta_{n}\right)-B_{i}+\bar{P}_{\kappa}\left(\tilde{\mathbf{X}}_{i}\right)^{\prime}\left(\theta-\theta_{\kappa 0}\right)-\bar{b}_{\kappa 0}\left(\tilde{\mathbf{X}}_{i}\right)\right]\right\} Z_{n i} K_{n i}, \\
\tilde{H}_{n}^{*}\left(\mathbf{b}, x^{1}, \theta\right) & =\left(n \delta_{n}\right)^{-1} \sum_{i=1}^{n}\left\{\alpha-F\left[Z_{n i}^{\prime}\left(\mathbf{b}-\beta_{n}\right)-B_{i}+\bar{P}_{\kappa}\left(\tilde{\mathbf{X}}_{i}\right)^{\prime}\left(\theta-\theta_{\kappa 0}\right)-\bar{b}_{\kappa 0}\left(\tilde{\mathbf{X}}_{i}\right) \mid \mathbf{X}_{i}\right]\right\} Z_{n i} K_{n i}, \\
\text { and } & \\
\Delta_{\tilde{H}_{n}}\left(\mathbf{b}, x^{1}, \theta\right) & =\tilde{H}_{n}\left(\mathbf{b}, x^{1}, \theta\right)-\tilde{H}_{n}^{*}\left(\mathbf{b}, x^{1}, \theta\right) .
\end{aligned}
$$


Then $\tilde{G}_{n}\left(\mathbf{b}, x^{1}\right)=\tilde{H}_{n}\left(\mathbf{b}, x^{1}, \hat{\theta}_{n \kappa}\right), \tilde{G}_{n}^{*}\left(\mathbf{b}, x^{1}\right)=\tilde{H}_{n}\left(\mathbf{b}, x^{1}, \hat{\theta}_{n \kappa}\right)$, and $\Delta_{\tilde{G}_{n}}\left(\mathbf{b}, x^{1}\right)=\Delta_{\tilde{H}_{n}}\left(\mathbf{b}, x^{1}, \hat{\theta}_{n \kappa}\right)$. The lemma follows if one can show that

$$
\sup _{\left\|\theta-\theta_{\kappa 0}\right\| \leq C[\kappa / n]^{1 / 2}}\left\|\Delta_{\tilde{H}_{n}}\left(\mathbf{b}, x^{1}, \theta\right)-\Delta_{G_{n}}\left(\mathbf{b}, x^{1}\right)\right\|=O_{p}\left[\left(n \delta_{n}\right)^{-1 / 2}\left[\kappa^{2} / n\right]^{1 / 4}(\log n)^{1 / 2}\right]
$$

for any $\mathbf{b}$ satisfying $\left\|\mathbf{b}-\beta_{n}\right\| \leq C\left(n \delta_{n}\right)^{-1 / 2}$. This can be proved by using virtually the same arguments as in the proofs of Lemmas A.5 and A.11.

Define

$$
Q_{n}=\left(n \delta_{n}\right)^{-1} \sum_{i=1}^{n} f\left(0 \mid \mathbf{X}_{i}\right) Z_{n i} Z_{n i}^{\prime} K_{n i} .
$$

Lemma A.13. As $n \rightarrow \infty$, for any $x^{1}$ such that $\left|x^{1}\right| \leq 1-\delta_{n}$,

$$
\tilde{G}_{n}^{*}\left(\hat{\mathbf{b}}_{n}, x^{1}\right)=-Q_{n}\left(\hat{\mathbf{b}}_{n}-\beta_{n}\right)+O_{p}\left(\delta_{n}^{r}\right)+O_{p}\left[\left(\hat{\mathbf{b}}_{n}-\beta_{n}\right)^{2}\right]+o_{p}\left[\left(n \delta_{n}\right)^{-1 / 2}\right] .
$$

Proof. Let $\tilde{\Delta}_{i}\left(\hat{\mathbf{b}}_{n}, x^{1}\right)=Z_{n i}^{\prime}\left(\hat{\mathbf{b}}_{n}-\beta_{n}\right)-B_{i}+\bar{P}_{\kappa}\left(\tilde{\mathbf{X}}_{i}\right)^{\prime}\left(\hat{\theta}_{n \kappa}-\theta_{\kappa 0}\right)-\bar{b}_{\kappa 0}\left(\tilde{\mathbf{X}}_{i}\right)$. A first-order Taylor expansion of $F\left[\tilde{\Delta}_{i}\left(\hat{\mathbf{b}}_{n}, x^{1}\right) \mid \mathbf{X}_{i}\right]$ gives

$$
\begin{aligned}
\tilde{G}_{n}^{*}\left(\hat{\mathbf{b}}_{n}, x^{1}\right) & =-\left(n \delta_{n}\right)^{-1} \sum_{i=1}^{n} \tilde{\Delta}_{i}\left(\hat{\mathbf{b}}_{n}, x^{1}\right) f\left(0 \mid \mathbf{X}_{i}\right) Z_{n i} K_{n i} \\
& -\left(n \delta_{n}\right)^{-1} \sum_{i=1}^{n} \tilde{\Delta}_{i}\left(\hat{\mathbf{b}}_{n}, x^{1}\right)\left[f\left(\tilde{\Delta}_{i}^{*}\left(\hat{\mathbf{b}}_{n}, x^{1}\right) \mid \mathbf{X}_{i}\right)-f\left(0 \mid \mathbf{X}_{i}\right)\right] Z_{n i} K_{n i} \\
& \equiv \tilde{G}_{n 1}^{*}\left(\hat{\mathbf{b}}_{n}\right)+\tilde{G}_{n 2}^{*}\left(\hat{\mathbf{b}}_{n}\right),
\end{aligned}
$$

where $\tilde{\Delta}_{i}^{*}\left(\hat{\mathbf{b}}_{n}, x^{1}\right)$ is between 0 and $\tilde{\Delta}_{i}\left(\hat{\mathbf{b}}_{n}, x^{1}\right)$.

Write $\tilde{G}_{n 1}^{*}\left(\hat{\mathbf{b}}_{n}\right)$ further as

$$
\tilde{G}_{n 1}^{*}\left(\hat{\mathbf{b}}_{n}\right)=\tilde{G}_{n 11}^{*}+\tilde{G}_{n 12}^{*}+\tilde{G}_{n 13}^{*}+\tilde{G}_{n 14}^{*},
$$

where

$$
\begin{aligned}
& \tilde{G}_{n 11}^{*}=-\left(n \delta_{n}\right)^{-1} \sum_{i=1}^{n} Z_{n i}^{\prime}\left(\hat{\mathbf{b}}_{n}-\beta_{n}\right) f\left(0 \mid \mathbf{X}_{i}\right) Z_{n i} K_{n i}, \\
& \tilde{G}_{n 12}^{*}=\left(n \delta_{n}\right)^{-1} \sum_{i=1}^{n} B_{i} f\left(0 \mid \mathbf{X}_{i}\right) Z_{n i} K_{n i}, \\
& \tilde{G}_{n 13}^{*}=-\left(n \delta_{n}\right)^{-1} \sum_{i=1}^{n} \bar{P}_{\kappa}\left(\tilde{\mathbf{X}}_{i}\right)^{\prime}\left(\hat{\theta}_{n \kappa}-\theta_{\kappa 0}\right) f\left(0 \mid \mathbf{X}_{i}\right) Z_{n i} K_{n i},
\end{aligned}
$$

and

$$
\tilde{G}_{n 14}^{*}=\left(n \delta_{n}\right)^{-1} \sum_{i=1}^{n} \bar{b}_{\kappa 0}\left(\tilde{\mathbf{X}}_{i}\right) f\left(0 \mid \mathbf{X}_{i}\right) Z_{n i} K_{n i}
$$


The first term is $\tilde{G}_{n 11}^{*}=-Q_{n}\left(\hat{\mathbf{b}}_{n}-\beta_{n}\right)$. Next consider the second term $\tilde{G}_{n 12}^{*}$. Notice that $\max _{1 \leq i \leq n}\left|B_{i}\right| \leq C \delta_{n}^{r}$ since $m_{1}$ is continuously $r$-times differentiable. Also, it is easy to see that

$$
\left\|\left(n \delta_{n}\right)^{-1} \sum_{i=1}^{n} f\left(0 \mid \mathbf{X}_{i}\right) Z_{n i} K_{n i}\right\|=O_{p}(1) .
$$

Therefore,

$$
\left\|\tilde{G}_{n 12}^{*}\right\|=O_{p}\left(\max _{1 \leq i \leq n}\left|B_{i}\right|\right)=O_{p}\left(\delta_{n}^{r}\right) .
$$

Now consider the third term $\tilde{G}_{n 13}^{*}$ in (A.11). Using Theorem 4.2, we have

$$
\begin{aligned}
\tilde{G}_{n 13}^{*} & =-\left(n^{2} \delta_{n}\right)^{-1} \sum_{i=1}^{n} \bar{P}_{\kappa}\left(\tilde{\mathbf{X}}_{i}\right)^{\prime} \Phi_{\kappa}^{-1} P_{\kappa}\left(\mathbf{X}_{j}\right)\left\{\alpha-1\left[U_{j} \leq 0\right]\right\} f\left(0 \mid \mathbf{X}_{i}\right) Z_{n i} K_{n i} \\
& -\left(n \delta_{n}\right)^{-1} \sum_{i=1}^{n} \bar{P}_{\kappa}\left(\tilde{\mathbf{X}}_{i}\right)^{\prime} \bar{B}_{n \kappa} f\left(0 \mid \mathbf{X}_{i}\right) Z_{n i} K_{n i} \\
& -\left(n \delta_{n}\right)^{-1} \sum_{i=1}^{n} \bar{P}_{\kappa}\left(\tilde{\mathbf{X}}_{i}\right)^{\prime} R_{n} f\left(0 \mid \mathbf{X}_{i}\right) Z_{n i} K_{n i} \\
& \equiv \tilde{G}_{n 131}^{*}+\tilde{G}_{n 132}^{*}+\tilde{G}_{n 133}^{*},
\end{aligned}
$$

where the remainder term $R_{n}$ is defined in Theorem 4.2 and

$$
\bar{B}_{n \kappa}=n^{-1} \sum_{j=1}^{n} \Phi_{\kappa}^{-1} f\left(0 \mid \mathbf{X}_{j}\right) P_{\kappa}\left(\mathbf{X}_{j}\right) b_{\kappa 0}\left(\mathbf{X}_{j}\right) .
$$

First, consider $\tilde{G}_{n 131}^{*}$. To show that

$$
\left\|\tilde{G}_{n 131}^{*}\right\|=o_{p}\left[\left(n \delta_{n}\right)^{-1 / 2}\right],
$$

define, for $k=1, \ldots, r$,

$$
g_{n}^{(k)}=\sum_{j=1}^{n} a_{j}^{(k)}\left\{\alpha-1\left[U_{j} \leq 0\right]\right\}
$$

where

$$
\begin{aligned}
a_{j}^{(k)} & =-n^{-3 / 2} \delta_{n}^{-1 / 2} \sum_{i=1}^{n} \bar{P}_{\kappa}\left(\tilde{\mathbf{X}}_{i}\right)^{\prime} \Phi_{\kappa}^{-1} P_{\kappa}\left(\mathbf{X}_{j}\right) f\left(0 \mid \mathbf{X}_{i}\right) Z_{n i}^{(k)} K_{n i} \\
& \equiv-n^{-3 / 2} \delta_{n}^{-1 / 2} \sum_{i=1}^{n} A_{i j}^{(k)} K_{n i}
\end{aligned}
$$

and $Z_{n i}^{(k)}$ is the $k$-th component of $Z_{n i}$. Then the $k$-th component of $\tilde{G}_{n 131}^{*}$ is $\left(n \delta_{n}\right)^{-1 / 2} g_{n}^{(k)}$ for $k=1, \ldots, r$. Therefore, to prove (A.12), it suffices to show that $g_{n}^{(k)}=o_{p}(1)$ for $k=1, \ldots, r$. Notice that $E\left[g_{n}^{(k)} \mid \mathbf{X}_{1}, \ldots, \mathbf{X}_{n}\right]=0$ and $\operatorname{Var}\left[g_{n}^{(k)} \mid \mathbf{X}_{1}, \ldots, \mathbf{X}_{n}\right] \leq C \sum_{j=1}^{n}\left[a_{j}^{(k)}\right]^{2}$. Hence,

$$
\max _{1 \leq j \leq n}\left|a_{j}^{(k)}\right|=o_{p}\left(n^{-1 / 2}\right)
$$


implies that $g_{n}^{(k)}=o_{p}(1)$. This equation (A.13) can be proved using arguments similar to those used in the proof of Lemma 7 of HM. The proof of (A.13) will be given at the end of the appendix.

Next consider $\tilde{G}_{n 132}^{*}$. By $K_{n i}=1\left(\left|x^{1}-X_{i}^{1}\right| \leq \delta_{n}\right) K_{n i}$ and the Schwarz inequality,

$$
\begin{aligned}
\left\|\tilde{G}_{n 132}^{*}\right\| & \leq\left[\left(n \delta_{n}\right)^{-1} \sum_{i=1}^{n}\left\{\bar{P}_{\kappa}\left(\tilde{\mathbf{X}}_{i}\right)^{\prime} \bar{B}_{n \kappa} 1\left(\left|x^{1}-X_{i}^{1}\right| \leq \delta_{n}\right)\right\}^{2}\right]^{1 / 2} \\
& \times\left[\left(n \delta_{n}\right)^{-1} \sum_{i=1}^{n}\left\|f\left(0 \mid \mathbf{X}_{i}\right) Z_{n i} K_{n i}\right\|^{2}\right]^{1 / 2}
\end{aligned}
$$

By the standard methods for bounding kernel estimators,

$$
\left(n \delta_{n}\right)^{-1} \sum_{i=1}^{n}\left\|f\left(0 \mid \mathbf{X}_{i}\right) Z_{n i} K_{n i}\right\|^{2}=O_{p}(1) .
$$

Also,

$$
\begin{gathered}
\left\|\left(n \delta_{n} f_{X^{1}}\left(x^{1}\right)\right)^{-1} \sum_{i=1}^{n} \bar{P}_{\kappa}\left(\tilde{\mathbf{X}}_{i}\right) \bar{P}_{\kappa}\left(\tilde{\mathbf{X}}_{i}\right)^{\prime} 1\left(\left|x^{1}-X_{i}^{1}\right| \leq \delta_{n}\right)-E\left[\bar{P}_{\kappa}(\tilde{\mathbf{X}}) \bar{P}_{\kappa}(\tilde{\mathbf{X}})^{\prime} \mid X^{1}=x^{1}\right]\right\|^{2} \\
=O_{p}\left(\kappa^{2} / n+\kappa^{2} \delta_{n}^{4}\right)=o_{p}(1),
\end{gathered}
$$

where $f_{X^{1}}\left(x^{1}\right)$ is the density of $X^{1}$. Then by (A.16) and Assumption 4.10, the largest eigenvalue of the first term inside $\|\cdot\|$ in (A.16) is bounded for all sufficiently large $n$. It follows that

$$
\begin{aligned}
& \left(n \delta_{n}\right)^{-1} \sum_{i=1}^{n}\left\{\bar{P}_{\kappa}\left(\tilde{\mathbf{X}}_{i}\right)^{\prime} \bar{B}_{n \kappa} 1\left(\left|x^{1}-X_{i}^{1}\right| \leq \delta_{n}\right)\right\}^{2} \\
& \leq C \bar{B}_{n \kappa}^{\prime}\left[\left(n \delta_{n} f_{X^{1}}\left(x^{1}\right)\right)^{-1} \sum_{i=1}^{n} \bar{P}_{\kappa}\left(\tilde{\mathbf{X}}_{i}\right) \bar{P}_{\kappa}\left(\tilde{\mathbf{X}}_{i}\right)^{\prime} 1\left(\left|x^{1}-X_{i}^{1}\right| \leq \delta_{n}\right)\right] \bar{B}_{n \kappa} \\
& \leq C\left\|\bar{B}_{n \kappa}\right\|^{2}
\end{aligned}
$$

for all sufficiently large $n$. In view of Lemma A.8, it can be shown that $\left\|\bar{B}_{n \kappa}\right\|=O\left(\kappa^{-r}\right)$. Therefore, by (A.14),

$$
\left\|\tilde{G}_{n 132}^{*}\right\| \leq O_{p}\left(\kappa^{-r}\right) O_{p}(1)=o_{p}\left[\left(n \delta_{n}\right)^{-1 / 2}\right]
$$

provided that $\delta_{n} \propto n^{-1 /(2 r+1)}$ and $n^{r /(2 r+1)} \kappa^{-r} \rightarrow 0$. In particular, if $\kappa \propto n^{\nu}$, then

$$
\nu>\frac{1}{2 r+1} .
$$

Now consider $\tilde{G}_{n 133}^{*}$. Arguments identical to those used to prove (A.17) gives

$$
\left\|\tilde{G}_{n 133}^{*}\right\| \leq O_{p}\left(\left\|R_{n}\right\|\right) O_{p}(1)=O_{p}\left[\left(\kappa^{2} / n\right)^{3 / 4}(\log n)^{1 / 2}+\kappa^{3 / 2} / n\right]=o_{p}\left[\left(n \delta_{n}\right)^{-1 / 2}\right]
$$


provided that $\delta_{n} \propto n^{-1 /(2 r+1)}$ and $\kappa n^{-(2 r+3) /(12 r+6)}(\log n)^{1 / 3} \rightarrow 0$. In particular, if $\kappa \propto n^{\nu}$, then

$$
\nu<\frac{2 r+3}{12 r+6}
$$

Combining (A.19) with (A.18) gives

$$
\frac{1}{2 r+1}<\nu<\frac{2 r+3}{12 r+6}
$$

which requires that $r$ must be larger than or equal to 2 . Combining the results for $\tilde{G}_{n 13 k}^{*}$ for $k=1,2,3$ gives

$$
\tilde{G}_{n 13}^{*}=o_{p}\left[\left(n \delta_{n}\right)^{-1 / 2}\right] .
$$

Next consider the fourth term $\tilde{G}_{n 14}^{*}$ in (A.11). Notice that

$$
\begin{aligned}
\left\|\tilde{G}_{n 14}^{*}\right\| & \leq\left[\left(n \delta_{n}\right)^{-1} \sum_{i=1}^{n}\left\|f\left(0 \mid \mathbf{X}_{i}\right) Z_{n i} K_{n i}\right\|\right] O\left(\kappa^{-r}\right) \\
& =O_{p}(1) O\left(\kappa^{-r}\right) \\
& =o_{p}\left[\left(n \delta_{n}\right)^{-1 / 2}\right] .
\end{aligned}
$$

Therefore, combining the results for $\tilde{G}_{n 1 k}^{*}$ for $k=1,2,3,4$ gives

$$
\tilde{G}_{n 1}^{*}\left(\hat{\mathbf{b}}_{n}\right)=-Q_{n}\left(\hat{\mathbf{b}}_{n}-\beta_{n}\right)+O_{p}\left(\delta_{n}^{r}\right)+o_{p}\left[\left(n \delta_{n}\right)^{-1 / 2}\right] .
$$

Now consider $\tilde{G}_{n 2}^{*}\left(\hat{\mathbf{b}}_{n}\right)$. It follows from Assumption 4.3 and Theorem 4.1 (b) that

$$
\begin{aligned}
\tilde{G}_{n 2}^{*}\left(\hat{\mathbf{b}}_{n}\right) & =O_{p}\left[\left\{\tilde{\Delta}_{i}\left(x^{1}, \hat{\mathbf{b}}_{n}\right)\right\}^{2}\right] \\
& =O_{p}\left[\left(\hat{\mathbf{b}}_{n}-\beta_{n}\right)^{2}+\delta_{n}^{2 r}+k^{2} / n+\kappa^{-2 r}\right] \\
& =O_{p}\left[\left(\hat{\mathbf{b}}_{n}-\beta_{n}\right)^{2}\right]+o_{p}\left[\left(n \delta_{n}\right)^{-1 / 2}\right] .
\end{aligned}
$$

Then the lemma follows from combining the results for $\tilde{G}_{n k}^{*}\left(\hat{\mathbf{b}}_{n}\right)$ for $k=1,2$.

Proof of Theorem 4.3. Part (a) can be proved using arguments identical to those used in the proof of Theorem 4.1 (a) with Lemmas A.9-A.13.

To prove part (b), write

$$
\begin{aligned}
\tilde{G}_{n}\left(\hat{\mathbf{b}}_{n}, x^{1}\right) & =\Delta_{G_{n}}\left(\beta_{n}, x^{1}\right)+\left[\Delta_{G_{n}}\left(\hat{\mathbf{b}}_{n}, x^{1}\right)-\Delta_{G_{n}}\left(\beta_{n}, x^{1}\right)\right] \\
& +\left[\Delta_{\tilde{G}_{n}}\left(\hat{\mathbf{b}}_{n}, x^{1}\right)-\Delta_{G_{n}}\left(\hat{\mathbf{b}}_{n}, x^{1}\right)\right]+\tilde{G}_{n}^{*}\left(\hat{\mathbf{b}}_{n}, x^{1}\right) .
\end{aligned}
$$

Combining Lemmas A.9, A.11 - A.13, and (A.21) with part (a) of the theorem gives

$$
Q_{n}\left(\hat{\mathbf{b}}_{n}-\beta_{n}\right)=G_{n}\left(\beta_{n}, x^{1}\right)-G_{n}^{*}\left(\beta_{n}, x^{1}\right)+\tilde{G}_{n 12}^{*}+r_{n 1},
$$


where $\tilde{G}_{n 12}^{*}$ was defined in the proof of Lemma A.13, and the remainder term $r_{n 1}$ satisfies $\left\|r_{n 1}\right\|=$ $o_{p}\left[\left(n \delta_{n}\right)^{-1 / 2}\right]$. By a first-order Taylor expansion and Assumption (4.3), it is easy to show that

$$
\left\|\tilde{G}_{n 12}^{*}-G_{n}^{*}\left(\beta_{n}, x^{1}\right)\right\|=O_{p}\left(\left\{\max _{1 \leq i \leq n}\left|B_{i}\right|\right\}^{2}\right)=O_{p}\left(\delta_{n}^{2 r}\right)=o_{p}\left[\left(n \delta_{n}\right)^{-1 / 2}\right] .
$$

Therefore, it follows that

$$
\left\|\hat{\mathbf{b}}_{n}-\beta_{n}-Q_{n}^{-1} G_{n}\left(\beta_{n}\right)\right\|=o_{p}\left[\left(n \delta_{n}\right)^{-1 / 2}\right]
$$

for all sufficiently large $n$. Furthermore, methods similar to those used to establish asymptotic properties of kernel estimators give $\left\|Q_{n}^{*}-Q^{*}\right\|=o_{p}(1)$, where

$$
\begin{aligned}
Q_{*} & =\int_{\tilde{\mathbf{x}} \in[-1,1]^{d-1}} f\left(0 \mid x^{1}, \tilde{\mathbf{x}}\right) f_{\mathbf{X}}\left(x^{1}, \tilde{\mathbf{x}}\right) d \tilde{\mathbf{x}} \int_{-1}^{1}\left(\begin{array}{cccc}
1 & u & \ldots & u^{r-1} \\
& & \ldots & \\
u^{r-1} & u^{r} & \ldots & u^{2(r-1)}
\end{array}\right) K(u) d u \\
& =\left[f_{1}\left(0 \mid x^{1}\right) f_{X^{1}}\left(x^{1}\right)\right] S(K) .
\end{aligned}
$$

Then it follows that

$$
\hat{m}_{1}\left(x^{1}\right)-m_{1}\left(x^{1}\right)=e_{1}^{\prime}\left(\hat{\mathbf{b}}_{n}-\beta_{n}\right)=e_{1}^{\prime} Q_{*}^{-1} G_{n}\left(\beta_{n}\right)+r_{n 2}
$$

where the remainder term $r_{n 2}$ satisfies $\left\|r_{n 2}\right\|=o_{p}\left[\left(n \delta_{n}\right)^{-1 / 2}\right]$.

Recall that $e_{1}^{\prime} S(K)^{-1}\left(1, u, \ldots, u^{r-1}\right)^{\prime} K(u)$ is a kernel of order $r$. Then parts (b) and (c) can be proved by using arguments identical to those used to establish asymptotic normality of local polynomial estimators.

Proof of (A.13). To show (A.13), first notice that

$$
\begin{aligned}
a_{j}^{(k)} & =-n^{-3 / 2} \delta_{n}^{-1 / 2} A_{j j}^{(k)} K_{n j}-n^{-3 / 2} \delta_{n}^{-1 / 2} \sum_{i=1, i \neq j}^{n} A_{i j}^{(k)} K_{n i} \\
& =-n^{-3 / 2} \delta_{n}^{-1 / 2} \sum_{i=1, i \neq j}^{n} A_{i j}^{(k)} K_{n i}+O_{p}\left(n^{-3 / 2} \delta_{n}^{-1 / 2} \zeta_{\kappa}^{2}\right) \\
& =-n^{-3 / 2} \delta_{n}^{-1 / 2} \sum_{i=1, i \neq j}^{n} A_{i j}^{(k)} K_{n i}+o_{p}\left(n^{-1 / 2}\right)
\end{aligned}
$$

uniformly over $j$. Write

$$
\begin{aligned}
& n^{-3 / 2} \delta_{n}^{-1 / 2} \sum_{i=1, i \neq j}^{n} A_{i j}^{(k)} K_{n i} \\
& =n^{-3 / 2} \delta_{n}^{-1 / 2} \sum_{i=1, i \neq j}^{n} E\left[A_{i j}^{(k)} \mid \mathbf{X}_{i}\right] K_{n i}+n^{-3 / 2} \delta_{n}^{-1 / 2} \sum_{i=1, i \neq j}^{n}\left\{A_{i j}^{(k)}-E\left[A_{i j}^{(k)} \mid \mathbf{X}_{i}\right]\right\} K_{n i} \\
& \equiv a_{j 1}^{(k)}+a_{j 2}^{(k)} .
\end{aligned}
$$


By $E\left[P_{\kappa}\left(\mathbf{X}_{j}\right) \mid \mathbf{X}_{i}\right]=E\left[P_{\kappa}\left(\mathbf{X}_{j}\right)\right]$ for $j \neq i, K_{n i}=1\left(\left|x^{1}-X_{i}^{1}\right| \leq \delta_{n}\right) K_{n i}$, and the Schwarz inequality,

$$
\begin{aligned}
\left|a_{j 1}^{(k)}\right| & \leq n^{-3 / 2} \delta_{n}^{-1 / 2} \sum_{i=1, i \neq j}^{n}\left|\bar{P}_{\kappa}\left(\tilde{\mathbf{X}}_{i}\right)^{\prime} \Phi_{\kappa}^{-1} E\left[P_{\kappa}\left(\mathbf{X}_{j}\right)\right] f\left(0 \mid \mathbf{X}_{i}\right) Z_{n i}^{(k)} K_{n i}\right| \\
& \leq n^{-1 / 2} \delta_{n}^{1 / 2}\left(\left(n \delta_{n}\right)^{-1} \sum_{i=1, i \neq j}^{n}\left\{\bar{P}_{\kappa}\left(\tilde{\mathbf{X}}_{i}\right)^{\prime} \Phi_{\kappa}^{-1} E\left[P_{\kappa}\left(\mathbf{X}_{j}\right)\right] 1\left(\left|x^{1}-X_{i}^{1}\right| \leq \delta_{n}\right)\right\}^{2}\right)^{1 / 2} \\
& \times\left(\left(n \delta_{n}\right)^{-1} \sum_{i=1, i \neq j}^{n}\left\{f\left(0 \mid \mathbf{X}_{i}\right) Z_{n i}^{(k)} K_{n i}\right\}^{2}\right)^{1 / 2} .
\end{aligned}
$$

As in (A.15) and (A.16),

$$
\left(n \delta_{n}\right)^{-1} \sum_{i=1, i \neq j}^{n}\left\{f\left(0 \mid \mathbf{X}_{i}\right) Z_{n i}^{(k)} K_{n i}\right\}^{2}=O_{p}(1)
$$

and

$$
\begin{gathered}
\left\|\left(n \delta_{n} f_{X^{1}}\left(x^{1}\right)\right)^{-1} \sum_{i=1, i \neq j}^{n} \bar{P}_{\kappa}\left(\tilde{\mathbf{X}}_{i}\right) \bar{P}_{\kappa}\left(\tilde{\mathbf{X}}_{i}\right)^{\prime} 1\left(\left|x^{1}-X_{i}^{1}\right| \leq \delta_{n}\right)-E\left[\bar{P}_{\kappa}(\tilde{\mathbf{X}}) \bar{P}_{\kappa}(\tilde{\mathbf{X}})^{\prime} \mid X^{1}=x^{1}\right]\right\|^{2} \\
=O_{p}\left(\kappa^{2} / n+\kappa^{2} \delta_{n}^{4}\right)=o_{p}(1) .
\end{gathered}
$$

Then by (A.25) and Assumption 4.10, the largest eigenvalue of the first term inside $\|\cdot\|$ in (A.25) is bounded for all sufficiently large $n$. Furthermore, in view of Assumption 4.6 (c), elements of $E\left[P_{\kappa}\left(\mathbf{X}_{j}\right)\right]$ are the Fourier coefficients of the density of $\mathbf{X}$. Since the density of $\mathbf{X}$ is bounded, $E\left[P_{\kappa}\left(\mathbf{X}_{j}\right)\right]^{\prime} E\left[P_{\kappa}\left(\mathbf{X}_{j}\right)\right]$ converges as $\kappa \rightarrow \infty$. Combining all these gives

$$
\begin{aligned}
& \left(n \delta_{n}\right)^{-1} \sum_{i=1, i \neq j}^{n}\left\{\bar{P}_{\kappa}\left(\tilde{\mathbf{X}}_{i}\right)^{\prime} \Phi_{\kappa}^{-1} E\left[P_{\kappa}\left(\mathbf{X}_{j}\right)\right] 1\left(\left|x^{1}-X_{i}^{1}\right| \leq \delta_{n}\right)\right\}^{2} \\
& \leq C E\left[P_{\kappa}\left(\mathbf{X}_{j}\right)\right]^{\prime} \Phi_{\kappa}^{-1}\left[\left(n \delta_{n} f_{X^{1}}\left(x^{1}\right)\right)^{-1} \sum_{i=1, i \neq j}^{n} \bar{P}_{\kappa}\left(\tilde{\mathbf{X}}_{i}\right) \bar{P}_{\kappa}\left(\tilde{\mathbf{X}}_{i}\right)^{\prime} 1\left(\left|x^{1}-X_{i}^{1}\right| \leq \delta_{n}\right)\right] \Phi_{\kappa}^{-1} E\left[P_{\kappa}\left(\mathbf{X}_{j}\right)\right] \\
& \leq C E\left[P_{\kappa}\left(\mathbf{X}_{j}\right)\right]^{\prime} E\left[P_{\kappa}\left(\mathbf{X}_{j}\right)\right] \\
& \leq C
\end{aligned}
$$

for all sufficiently large $n$, where the constant $C$ can be chosen uniformly over $j$. Combining this and (A.24) with (A.23) proves that $\max _{1 \leq j \leq n}\left|a_{j 1}^{(k)}\right|=o_{p}\left(n^{-1 / 2}\right)$. 
Now, it remains to prove that $\max _{1 \leq j \leq n}\left|a_{j 2}^{(k)}\right|=o_{p}\left(n^{-1 / 2}\right)$. To do so, notice that

$$
\begin{aligned}
E\left[\left|a_{j 2}^{(k)}\right|^{2} \mid\left\{\mathbf{X}_{i}\right\}_{i=1, i \neq j}^{n}\right] & =n^{-3} \delta_{n}^{-1} \sum_{i=1, i \neq j}^{n} \sum_{l=1, l \neq j}^{n} \bar{P}_{\kappa}\left(\tilde{\mathbf{X}}_{i}\right)^{\prime} \Phi_{\kappa}^{-1} \\
& \times E\left[\left\{P_{\kappa}\left(\mathbf{X}_{j}\right)-E\left[P_{\kappa}\left(\mathbf{X}_{j}\right)\right]\right\}\left\{P_{\kappa}\left(\mathbf{X}_{j}\right)-E\left[P_{\kappa}\left(\mathbf{X}_{j}\right)\right]\right\}^{\prime} \mid\left\{\mathbf{X}_{i}\right\}_{i=1, i \neq j}^{n}\right] \\
& \times \Phi_{\kappa}^{-1} \bar{P}_{\kappa}\left(\tilde{\mathbf{X}}_{l}\right) f\left(0 \mid \mathbf{X}_{i}\right) Z_{n i}^{(k)} K_{n i} f\left(0 \mid \mathbf{X}_{l}\right) Z_{n l}^{(k)} K_{n l} \\
& \leq C n^{-3} \delta_{n}^{-1} \sum_{i=1, i \neq j}^{n} \sum_{l=1, l \neq j}^{n} \bar{P}_{\kappa}\left(\tilde{\mathbf{X}}_{i}\right)^{\prime} \Phi_{\kappa}^{-1} \bar{P}_{\kappa}\left(\tilde{\mathbf{X}}_{l}\right) f\left(0 \mid \mathbf{X}_{i}\right) Z_{n i}^{(k)} K_{n i} f\left(0 \mid \mathbf{X}_{l}\right) Z_{n l}^{(k)} K_{n l} \\
& \leq C n^{-1} \delta_{n}\left[\left(n \delta_{n} f_{X^{1}}\left(x^{1}\right)\right)^{-1} \sum_{i=1, i \neq j}^{n} K_{n i} \bar{P}_{\kappa}\left(\tilde{\mathbf{X}}_{i}\right)^{\prime}\right] \Phi_{\kappa}^{-1} \\
& \times\left[\left(n \delta_{n} f_{X^{1}}\left(x^{1}\right)\right)^{-1} \sum_{l=1, l \neq j}^{n} \bar{P}_{\kappa}\left(\tilde{\mathbf{X}}_{l}\right) K_{n l}\right] \\
& \leq C n^{-1} \delta_{n} E\left[\bar{P}_{\kappa}(\tilde{\mathbf{X}}) \mid X^{1}=x^{1}\right]^{\prime} E\left[\bar{P}_{\kappa}(\tilde{\mathbf{X}}) \mid X^{1}=x^{1}\right]
\end{aligned}
$$

for all sufficiently large $n$. Elements of $E\left[\bar{P}_{\kappa}(\tilde{\mathbf{X}}) \mid X^{1}=x^{1}\right]$ are the Fourier coefficients of the conditional density of $\tilde{\mathbf{X}}$ given $X^{1}=x^{1}$, which is bounded. Hence, $E\left[\bar{P}_{\kappa}(\tilde{\mathbf{X}}) \mid X^{1}=x^{1}\right]^{\prime} E\left[\bar{P}_{\kappa}(\tilde{\mathbf{X}}) \mid X^{1}=x^{1}\right]$ converges as $\kappa \rightarrow \infty$, implying that

$$
E\left[\left|a_{j 2}^{(k)}\right|^{2} \mid\left\{\mathbf{X}_{i}\right\}_{i=1, i \neq j}^{n}\right]=o_{p}\left(n^{-1}\right)
$$

uniformly over $j$. This in turn implies that $\max _{1 \leq j \leq n}\left|a_{j 2}^{(k)}\right|=o_{p}\left(n^{-1 / 2}\right)$ by Markov inequality.

\section{References}

Bertail, P., D.N. Politis, and J.P. Romano (1999): On subsampling estimators with unknown rate of convergence, Journal of the American Statistical Association 94, 569579 .

Chaudhuri, P. (1991): Nonparametric estimates of regression quantiles and their local bahadur representation, Annals of Statistics 19, 760-777.

Chaudhuri, P., K. Doksum, \& A. Samarov (1997): On average derivative quantile regression. Annals of Statistics 25, 715-744.

De Gooijer, J.G. and D. Zerom (2003): On additive conditional quantiles with highdimensional covariates, Journal of the American Statistical Association, 98, 135-146.

Doksum, K. and J.-Y. Koo (2000): On spline estimators and prediction intervals in nonparametric regression, Computational Statistics $\&$ Data Analysis, 35, 76-82. 
Fan, J. and I. Gijbels (1996): Local Polynomial Modelling and Its Applications. London: Chapman and Hall.

Fan, J., T.C. Hu, and Y.K. Truong (1994): Robust non-parametric function estimation, Scandinavian Journal of Statistics 21, 433-446.

Gozalo, P.L. and O.B. Linton (2001): Testing additivity in generalized nonparametric regression models with estimated parameters, Journal of Econometrics, 104, 1-48.

Härdle, W. (1990): Applied nonparametric regression. Cambridge: Cambridge University Press.

Hastie, T.J. and R.J. Tibshirani (1990): Generalized Additive Models. London: Chapman and Hall.

He, X. and P. Shi (1994): Convergence rate of B-spline estimators of nonparametric conditional quantile functions, Journal of Nonparametric Statistics 3, 299-308.

He, X. and P. Shi (1996): Bivariate tensor-product B-splines in a partly linear model, Journal of Multivariate Analysis 58, 162-181.

He, X. and P. Shi (1998): Monotone B-spline smoothing, Journal of the American Statistical Association 93, 643-650.

He, X. and S. Portnoy (2000): Some asymptotic results on bivariate quantile splines, Journal of Statistical Planning and Inference, 91, 341-349.

Horowitz, J.L. (1993): Semiparametric estimation of a work-trip mode choice model, Journal of Econometrics, 58, 49-70.

Horowitz, J.L. and S. Lee (2002): Semiparametric methods in applied econometrics: do the models fit the data?, Statistical Modelling, 2, 3-22.

Horowitz, J.L. and E. Mammen (2004): Nonparametric estimation of an additive model with a link function, forthcoming, Annals of Statistics, 32, December.

Horowitz, J.L. and N.E. Savin (2001): Binary response models: logits, probits, and semiparametrics, Journal of Economic Perspectives, 15, 43-56.

Horowitz, J.L. and V.G. Spokoiny (2002): An adaptive, rate-optimal test of linearity for median regression models, Journal of the American Statistical Association, 97, 822835.

Hristache, M., A. Juditsky, and V. Spokoiny (2001): Direction estimation of the index coefficients in a single-index model, Annals of Statistics, 29, 595-623. 
Huang, J.Z. (2003): Local asymptotics for polynomial spline regression, Annals of Statistics, 31, 1600-1635.

Ichimura, H. (1993): Semiparametric least squares (SLS) and weighted SLS estimation of single-index models, Journal of Econometrics, 58, 71-120.

Khan, S. (2001): Two stage rank estimation of quantile index models, Journal of Econometrics, 100, 319-355.

Knight, K. (1998): Limiting distributions for $L_{1}$ regression estimators under general conditions, Annals of Statistics 26, 755-770.

Koenker, R. and G. Bassett (1978): Regression quantiles, Econometrica, 46, 33-50.

Lee, S. (2003): Efficient semiparametric estimation of a partially linear quantile regression model, Econometric Theory, 1-31.

Newey, W. K. (1997): Convergence rates and asymptotic normality for series estimators, Journal of Econometrics, 79, 147-168.

Portnoy, S. (1997): Local asymptotics for quantile smoothing splines, Annals of Statistics, $25,414-434$.

Powell, J. L., J. H. Stock, and T. M. Stoker (1989): Semiparametric estimation of index coefficients, Econometrica, 57, 1403-30.

Robinson, P. M. (1988): Root-N consistent semiparametric regression, Econometrica, 56, 931-954.

Ruppert, D. and M.P. Wand (1994): Multivariate locally weighted least squares estimation, Annals of Statistics, 22, 1346-1370.

van der Vaart, A. W. and J. A. Wellner (1996), Weak Convergence and Empirical Processes, New-York: Springer-Verlag.

Welsh, A. H. (1989): On M-processes and M-estimation, Annals of Statistics, 17, 337-361.

Yafeh, Y. and O. Yosha (2003): Large shareholders and Banks: Who monitors and how? Economic Journal, 113, 128-146. 
Table 1. Results of Monte Carlo Experiments

\begin{tabular}{|c|c|c|c|c|c|}
\hline \multirow{2}{*}{$\rho$} & \multirow[t]{2}{*}{$n$} & \multicolumn{2}{|c|}{ Results for $m_{1}$} & \multicolumn{2}{|c|}{ Results for $m_{2}$} \\
\hline & & MI & $2 \mathrm{~S}$ & MI & $2 \mathrm{~S}$ \\
\hline \multirow{3}{*}{0.2} & & & $d=2$ & & \\
\hline & 100 & 0.0834 & 0.0783 & 0.1430 & 0.1497 \\
\hline & 200 & 0.0560 & 0.0519 & 0.0964 & 0.1125 \\
\hline \multirow[t]{2}{*}{0.8} & 100 & 0.1638 & 0.0920 & 0.2957 & 0.1620 \\
\hline & 200 & 0.1331 & 0.0621 & 0.2602 & 0.1146 \\
\hline \multirow{3}{*}{0.2} & & & $d=5$ & & \\
\hline & 100 & 0.1268 & 0.0688 & 0.1914 & 0.1466 \\
\hline & 200 & 0.0917 & 0.0534 & 0.1293 & 0.1176 \\
\hline \multirow[t]{2}{*}{0.8} & 100 & 0.1810 & 0.0893 & 0.4060 & 0.1618 \\
\hline & 200 & 0.1650 & 0.0638 & 0.3578 & 0.1208 \\
\hline \multirow[t]{2}{*}{$\rho$} & \multirow[t]{2}{*}{$n$} & \multicolumn{2}{|c|}{ Results for $m_{1}$} & \multicolumn{2}{|c|}{ Results for $m_{2}$} \\
\hline & & $\mathrm{S}$ & $2 \mathrm{~S}$ & $\mathrm{~S}$ & $2 \mathrm{~S}$ \\
\hline \multirow{3}{*}{0.2} & & & $d=2$ & & \\
\hline & 100 & 0.0758 & 0.0708 & 0.1089 & 0.1104 \\
\hline & 200 & 0.0536 & 0.0492 & 0.0798 & 0.0803 \\
\hline \multirow[t]{2}{*}{0.8} & 100 & 0.0842 & 0.0798 & 0.1133 & 0.1153 \\
\hline & 200 & 0.0599 & 0.0557 & 0.0825 & 0.0834 \\
\hline
\end{tabular}

Note: The values shown in Table 1 are the average absolute deviation errors (AADE's) for the marginal integration (MI), spline (S) and two-stage (2S) estimators. 

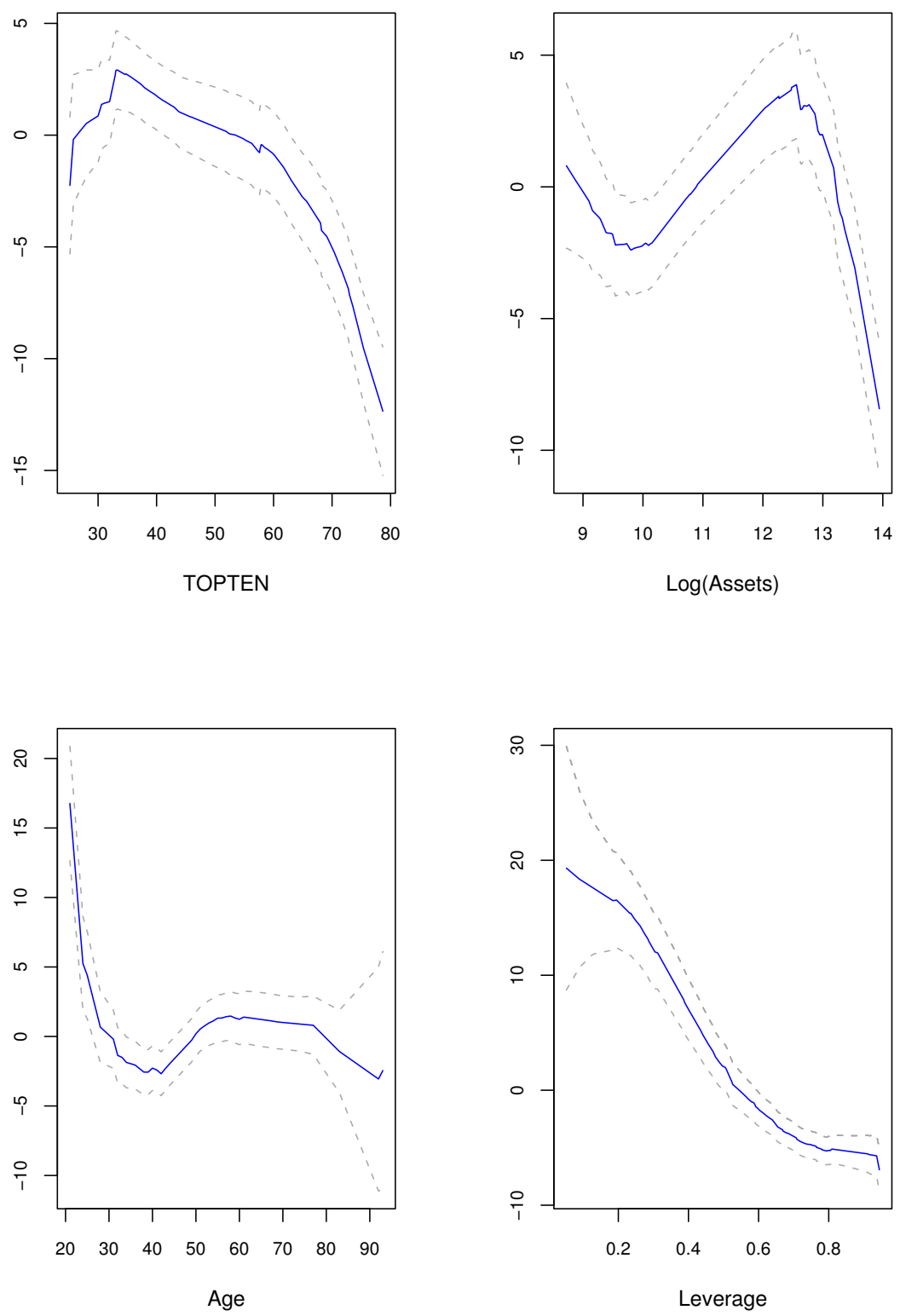

Figure 1. Estimation Results

Note: The estimated additive components of a nonparametric additive median regression model are shown along with their $90 \%$ pointwise confidence intervals. 

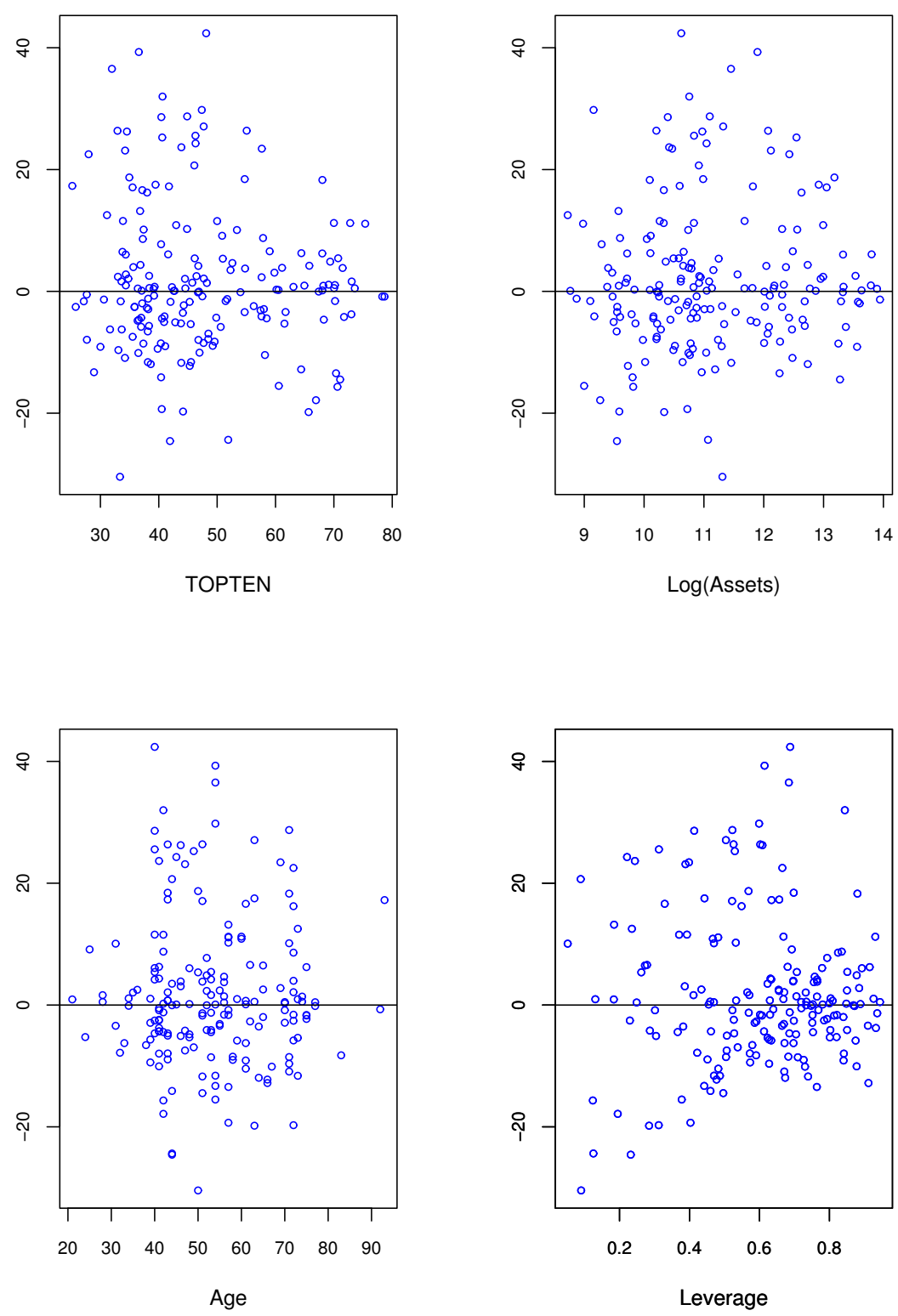

Figure 2. Residual Plots

Note: The estimated residuals are plotted against each of covariates. 